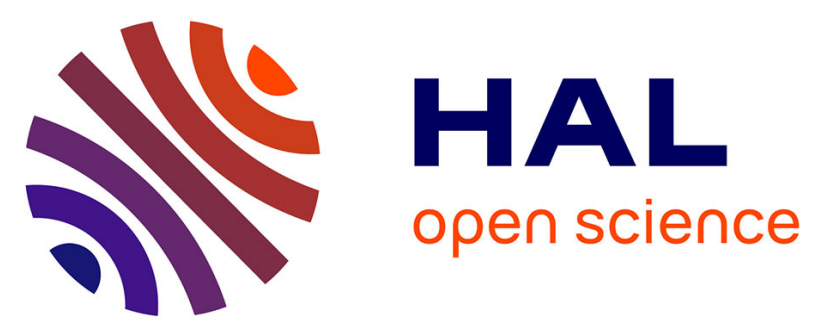

\title{
Rheology, force transmission, and shear instabilities in frictional granular media from biaxial numerical tests using the contact dynamics method
}

\author{
Alfredo Taboada, Kuo-Jen Chang, Farhang Radjai, Frédéric Bouchette
}

\section{To cite this version:}

Alfredo Taboada, Kuo-Jen Chang, Farhang Radjai, Frédéric Bouchette. Rheology, force transmission, and shear instabilities in frictional granular media from biaxial numerical tests using the contact dynamics method. Journal of Geophysical Research: Solid Earth, 2005, 110 (B9), pp.B09202. 10.1029/2003JB002955 . hal-00759654

\section{HAL Id: hal-00759654 https://hal.science/hal-00759654}

Submitted on 4 Jul 2017

HAL is a multi-disciplinary open access archive for the deposit and dissemination of scientific research documents, whether they are published or not. The documents may come from teaching and research institutions in France or abroad, or from public or private research centers.
L'archive ouverte pluridisciplinaire $\mathbf{H A L}$, est destinée au dépôt et à la diffusion de documents scientifiques de niveau recherche, publiés ou non, émanant des établissements d'enseignement et de recherche français ou étrangers, des laboratoires publics ou privés. 


\title{
Rheology, force transmission, and shear instabilities in frictional granular media from biaxial numerical tests using the contact dynamics method
}

\author{
Alfredo Taboada and Kuo-Jen Chang \\ Laboratoire de Dynamique de la Lithosphère, Université Montpellier II, Montpellier, France \\ Farhang Radjaï \\ Laboratoire de Mécanique et Genie Civil, Université Montpellier II, Montpellier, France
}

Frédéric Bouchette

Laboratoire de Dynamique de la Lithosphère, Université Montpellier II, Montpellier, France

Received 23 December 2003; revised 19 November 2004; accepted 22 February 2005; published 10 September 2005.

[1] By means of the contact dynamics discrete element method, we investigate the quasistatic behavior of granular media composed of rigid frictional particles. Eluding specific modeling of the contact rheology, this method is suitable for numerical simulation of the plastic deformations of granular materials. We studied the macroscopic stress-strain and volume-change behavior, as well as force transmission and shear instabilities, in a two-dimensional biaxial geometry for dense samples composed of 5000 rigid disks. The peak and residual strengths and shear bands were analyzed by varying the confining pressure and the coefficient of friction between particles. The results are consistent with well-known features of the plasticity of noncohesive granular media. The mechanical behavior is rigid-plastic governed by a Mohr-Coulomb yield criterion and showing strain hardening and softening. Conjugated shear bands characterize plastic failure. The volumetric strain is globally dilatant with considerable expansion observed along shear bands. The macroscopic coefficient of friction, determined from peak and residual strengths, increases nonlinearly and saturates to a constant value as a function of contact friction. The strong force chains are mostly parallel to the major principal stress axis, yet deviations are observed near the shear bands. These chains are often composed of particles that are larger than the average. The deviatoric stress shows small fluctuations often in the form of rapid falls that are correlated with tiny contractional events. This behavior is interpreted in terms of the propagation of dynamic shear instabilities along the shear bands, in close analogy with stick-slip behavior.

Citation: Taboada, A., K.-J. Chang, F. Radjaï, and F. Bouchette (2005), Rheology, force transmission, and shear instabilities in frictional granular media from biaxial numerical tests using the contact dynamics method, J. Geophys. Res., 110, B09202, doi:10.1029/2003JB002955.

\section{Introduction}

[2] Granular materials are commonly observed in natural deposits such as colluvium which consists of a poorly sorted mixture of angular rock fragments and fine-grained particles [Turner, 1996]. Particle sizes may range from huge boulders tens of meters long, detached from bedrock cliffs as a result of physical fragmentation processes, to rock flour generated by abrasive wear along frictional surfaces. Fault breccia and gouge observed in brittle shear zones are also significant examples of naturally occurring granular materials with specific mechanical properties that control processes such

Copyright 2005 by the American Geophysical Union. 0148-0227/05/2003JB002955\$09.00 as the surface propagation of seismic ruptures [Scholz, 1990; Mair et al., 2002].

[3] Geological landscapes are often shaped by dynamical surface processes involving granular materials [Friedmann et al., 2003]. Sand dunes result from the accumulation of sand grains moving by saltation and sliding along the slip face [Bagnold, 1941]. The sliding process involves small avalanches, triggered once the angle of repose is reached. Landslides often involve heterogeneous granular materials such as rocks and debris which may fall, topple or slide [Cruden and Varnes, 1996]. The complex kinematics of the flowing mass depends on the morphological and geological characteristics of the slope, as well as the rheology of surface materials and the triggering processes.

[4] The analysis of surface processes involving fragmented material in the geological context may benefit from 
the ongoing interdisciplinary research on granular materials. The mechanical behavior of noncohesive granular materials has been widely studied by experiments and numerical simulations in soil mechanics, in condensed matter physics and in different engineering applications such as powder technology. The strength and volume change properties of granular materials have been analyzed for various grain size distributions and boundary conditions [e.g., Cambou, 1993; Thornton and Sun, 1993; Oger et al., 1998; Morgan, 1999; Oda and Iwashita, 1999; Thornton and Zhang, 2001].

[5] New insights about the microscopic origins of the specific elastic and plastic properties of granular media have been obtained by a careful analysis of the microstructure [Oda, 1972a, 1972b; Rothenburg and Bathurst, 1989; Roux and Combe, 2002], displacement fields [Kuhn, 1999; Radjaï and Roux, 2002], stress transmission [Dantu, 1957; Cundall et al., 1982; Jaeger et al., 1996; Radjaï et al., 1998], and shear band formation [Vardoulakis et al., 1978; Bardet and Proubet, 1992; Desrues et al., 1996; Calvetti et al., 1997; Place and Mora, 2000].

[6] Since two decades, distinct element simulations have proved to be a powerful tool in the field of granular materials [Cundall and Stack, 1979; Jean, 2001]. The general philosophy of this approach is to integrate the equations of dynamics over small time steps for individual grains by taking into account the grain-grain interactions as well as the bulk and boundary forces. While the grains are assumed to be undeformable, the grain interactions are often modeled through normal (repulsion) and tangential (friction) forces depending elastically on the relative displacements at the contact point [Cundall and Stack, 1979; Luding, 1998; Oron and Herrmann, 1998]. Damping actions are introduced in order to ensure numerical stability. This approach requires very fine time stepping so as to resolve the evolution of the small overlaps between the grains. Such a fine resolution and other numerical precautions cannot be avoided when small elastic deformations of a granular material are of primary importance, e.g., for sound propagation. There are, however, two situations where this "soft-particle" approach is not suitable: (1) when the grains are very rigid or the confining stress is too low and (2) when one is interested in the plastic behavior occurring beyond elastic response times and for very large strains. Then, the natural physical approach is to rely on a "hard-particle" approximation. The numerical approach that is well suited to simulate multibody dynamics in this limit is the contact dynamics (CD) method. This method was initiated by Moreau [1988, 1994, 1999] and Jean [1995, 1999], and since then it has been used successfully by different authors for the investigation of granular materials [Moreau, 1993; Radjaï et al., 1996; Daudon et al., 1997; Chevoir et al., 2001; Staron et al., 2002].

[7] In this paper, we are interested in the quasi-static rheology of materials composed of perfectly rigid particles subjected to a classical test along a monotonic biaxial strain path and simulated by the CD method. The idea is to study the purely plastic and hardening-softening behavior of a granular material in the absence of elastic strains and cohesion. Plastic deformation refers to the irreversible strain that is not recovered upon unloading. The tests are carried out on numerical samples composed of 5000 rigid disks. The numerical device is comparable to a triaxial test apparatus, in which the sample is subjected to a confining pressure applied through a deformable membrane. The grain size distribution is uniform by weight, with sizes ranging between $1 \mathrm{~m}$ and $4 \mathrm{~m}$. The samples are compressed up to $10 \%$ of their initial height, allowing to estimate both their maximum and residual strengths. The mechanical behavior was analyzed for different values of the confining pressure and the coefficient of friction between particles, the total number of tests being 70 .

[8] We consider in detail the peak and residual stresses, volumetric strains, the influence of the bulk density, the formation of shear bands, the stress transmission, the influence of boundary conditions, and the intermittent dynamics at small strain increments. We analyze, in particular, the propagation of macroscopic shear bands and dynamic instabilities within the particle assembly for low and high friction coefficients. The stick-slip behavior along shear bands is described and interpreted in terms of the accumulation and release of potential energy. The shear band characteristics are related to deformation mechanisms observed at the particle scale. We also study the relationship between the contact friction and the macroscopic friction.

[9] It is noteworthy that the absence of grain elasticity in our calculations imparts a peculiar informative dimension to the results when they are compared to simulations with elastic contacts. For example, the presence of a stick-slip behavior in our simulations involves no stored elastic energy. In the same way, the initial quasi-linear increase of deviatoric stress with axial strain in our simulations is a purely hardening effect. On the other hand, through these studies, we illustrate the potential interest of the CD approach as an attractive investigation tool for many geological processes involving fragmented material. In particular, since no length scale is associated with the contacts, the time step may be quite large (e.g., $10^{-4} \mathrm{~s}$ ). Numerical approaches that consider contact forces involving a length scale, such as those generated by elastic spring interactions, usually require a much finer time step. Nonsmooth processes such as the initiation and propagation of shear bands and fractures are readily simulated; the absence of damping actions at the contacts allows one to observe fine timescaled mechanical processes such as shear instabilities.

[10] The main features of the CD method are introduced in section 2. Then, we present the characteristics of our numerical setup (boundary conditions, driving), the grain properties and the procedures of sample preparation. Finally, we present in several sections our results from the numerical tests.

\section{Contact Dynamics Method}

[11] In contrast to usual distinct element calculations used in the field of geomaterials, the $\mathrm{CD}$ method may be described as a "nonsmooth" approach in the sense that the dynamics is formulated in such a way that it deals with velocity jumps as well as with smooth velocity variations. In this framework, inelastic collisions and the Coulomb friction law can be tackled without resorting to elastic repulsive potentials or viscous-regularized force laws. This method was proposed and developed on a mathematical background of nonsmooth dynamics and concepts from convex analysis in order to calculate efficiently the me- 
chanical interactions within large particle sets [Moreau, 1988, 1994, 1999; Jean, 1995, 1999]. The equations of the CD method, as implemented for the simulations of the present study, are presented in some detail in Appendix A which is also meant to provide a didactic introduction to the method for the novice.

[12] The particles are rigid disks exerting normal and shear forces, $N$ and $T$, respectively, on each other at their contact points. In the absence of adhesion, the normal contact forces are compressive. We attribute a positive sign to compressive contact forces. The relative normal velocity $U^{n}$ between two particles in contact is considered as positive when they move away from each other (i.e., particles are at incipient separation). Then, the impenetrability of the particles implies the following "unilateral" conditions:

$$
\begin{array}{ll}
N \geq 0 & U^{n}=0 \\
N=0 & U^{n}>0 .
\end{array}
$$

On the other hand, the Coulomb friction law is expressed by a set of inequalities:

$$
\begin{array}{cc}
T=-\mu N & U^{t}>0, \\
-\mu N \leq T \leq \mu N & U^{t}=0, \\
T=\mu N & U^{t}<0,
\end{array}
$$

where $U^{t}$ is the relative tangential velocity at the contact and $\mu$ is the coefficient of friction.

[13] The unknown variables are particle velocities and contact forces. These are calculated at each time step by taking into account the conservation of momenta, the constraints expressed via inequalities (1) and (2), and the dissipation of kinetic energy during particle collisions, formulated in terms of normal and tangential restitution coefficients. Although the uniqueness of the solution is not guaranteed, the iterative research of the solution by means of a nonlinear Gauss-Seidel scheme converges always almost to the same solution, the difference between different solutions being of the order of the numerical precision. Physically, this means that the strength and plastic properties result from dissipative processes between the particles (dry friction and inelastic collisions) and group rearrangements, strongly constrained by the inequalities (1) and (2). These properties might depend only marginally on the deformability of the particles. The latter is assumed to be small compared to particle sizes also in the usual discrete element approach based on contact elasticity.

[14] Within the CD framework, it is also possible to introduce rate and state-dependent friction laws such as the well-known laws established first in rock mechanics [e.g., Dieterich, 1979; Ruina, 1983; Abe et al., 2002]. The influence of such fine physical parameters on multibody dynamics in granular media merits a separate investigation. For example, in the quasi-static limit, which is at the focus of this paper, contact aging might contribute to the shear strength. However, as we shall see in the following sections, collective phenomena and shear instabilities occur already in the presence of the most basic Coulombian friction described by the inequalities (2). This corresponds to a nonsmooth law in the sense that the relationship between the friction force $T$ and the sliding velocity $U^{t}$ cannot be reduced to a single-valued function. The same is true for the unilateral conditions expressed by inequalities (1). Such nonsmooth laws are readily implemented in the framework of the CD method, whereas their implementation in the usual discrete element approach requires a regularization scheme that transforms the set of inequalities into a set of equalities requiring at the same time some purely numerical parameters to be introduced.

[15] We see that the major challenge addressed in this paper is not the use of complex interaction laws, which remains necessary and feasible, but rather to be able to simulate the basic laws without introducing unphysical parameters. This is the main merit of the CD method which we exploit in the present studies prior to its application to more complex geological situations. In this respect, while the present study is entirely devoted to the rheology of rigid cohesionless particle sets, let us observe that both cohesion and contact deformation, as observed in rocks [e.g., Hazzard et al., 2000], can be modeled in the spirit of the CD method [Radjaï et al., 2001]. For example, the tensile contact strength can be introduced by specifying a negative (according to our sign conventions) threshold for normal contact forces or impulsions. Adding an offset to the tangential force threshold increases the shear strength. Contact deformation is introduced by specifying an "influence zone" around the contact points: Cohesive interactions are present as long as the contact points belong to this zone. We have tested this approach and our preliminary results are consistent with cohesive Mohr-Coulomb behavior.

\section{Numerical Procedures}

[16] We generate numerical samples composed of 5000 rigid circular particles in a two-dimensional space (also represented as hard disks on a planar surface) (Figure 1a). The initial sample is built by means of simple geometrical rules and then it is molded by means of a uniaxial compaction into a rectangular shape, before loading. Once compacted, the sample will be sheared biaxially under different confining pressures. The testing procedure consists of two different phases in analogy with experimental procedures used in soil mechanics biaxial and triaxial tests (Figures $1 \mathrm{~b}$ and 1c) [e.g., Bardet, 1997; Alshibli and Sture, 2000]. First, the sample is subjected to a uniform confining pressure along its boundaries. Then, it is shortened along the vertical direction at a constant rate.

\subsection{Material Parameters}

[17] The following parameters were used in all simulations:

[18] 1. In order to have a more homogeneous stress distribution, the gravity is set to zero.

[19] 2. The density of the particles is that of rock forming minerals in the crust (i.e., quartz): $\rho=2600 \mathrm{~kg} / \mathrm{m}^{3}$.

[20] 3. The time step is $\Delta \tau=0.0005 \mathrm{~s}$. The adequacy of the time step to simulate precisely the deformation process is evaluated as a function of the tolerated overlap between particles. The tolerated overlap in our simulations is below $1 \%$ the smallest particle size, whereas the mean overlap is generally one to two orders of magnitude lower. 

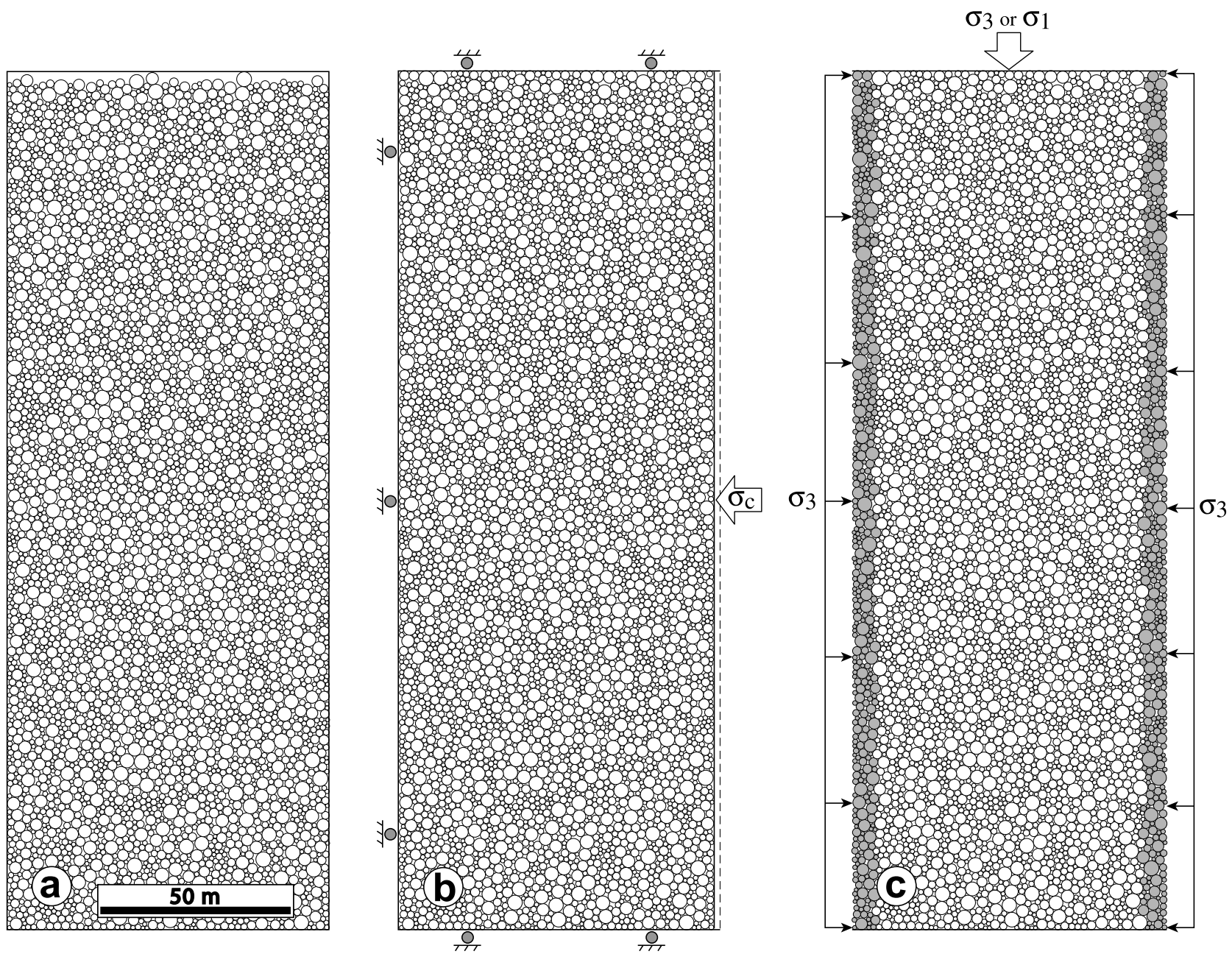

Figure 1. (a) Numerical sample composed of 5000 rigid disks, built by geometrical construction rules. (b) Uniaxial compaction of the numerical sample by means of a horizontal stress $\sigma_{c}$ applied on the right vertical wall. (c) Application of the confining pressure $\sigma_{3}$ and subsequent biaxial compression of the sample. The variable $\sigma_{3}$ is applied through deformable walls materialized by the gray disks in the vertical strips. The confining pressure is also exerted on the top horizontal wall, through an external vertical force. The biaxial compression is obtained by gradually increasing the axial displacement of the top horizontal wall at a constant rate. The variable $\sigma_{1}$ is the axial stress exerted by the disks on the top horizontal wall during shear.

[21] 4. The normal and tangential restitution coefficients are fixed to zero in order to reduce possible dynamic effects.

\subsection{Grain Size Distribution}

[22] Figure 2 displays the volume fraction of the particles below a given radius as a function of this radius. The particle radii are evenly distributed by weight (or by volume fraction) between $R_{\mathrm{Min}}=0.5 \mathrm{~m}$ and $R_{\mathrm{Max}}=2 \mathrm{~m}$. The choice of metric size particles is deliberate since one of our goals is to apply the CD method to simulate geological processes of hectometric to kilometric scales, such as rock avalanches (without particle rupture). These models would require less than 50,000 particles, which is roughly the maximum number of particles that can be simulated by a personal computer in a reasonable time (less than one day). Nevertheless, the set of equations defining contact forces are independent of particle sizes and do not involve a length scale. Thus our results would be analogous for homothetic

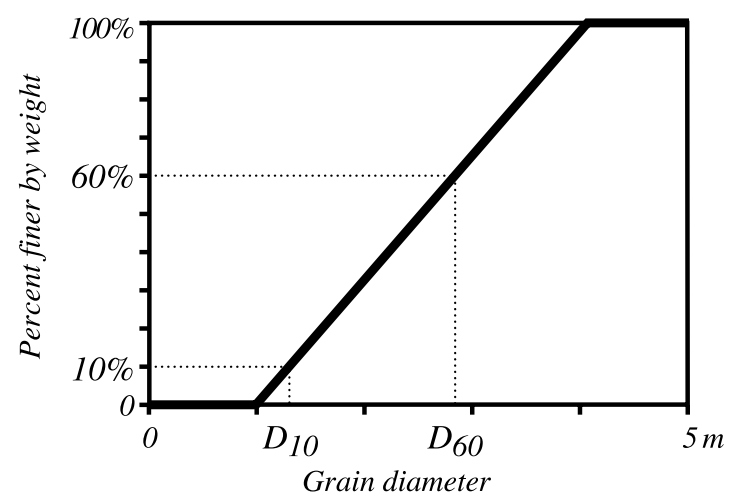

Figure 2. Grain size distribution of the numerical sample. Particle radii are evenly distributed by weight between $R_{\text {Min }}=0.5 \mathrm{~m}$ and $R_{\text {Max }}=2 \mathrm{~m}$, respectively. 


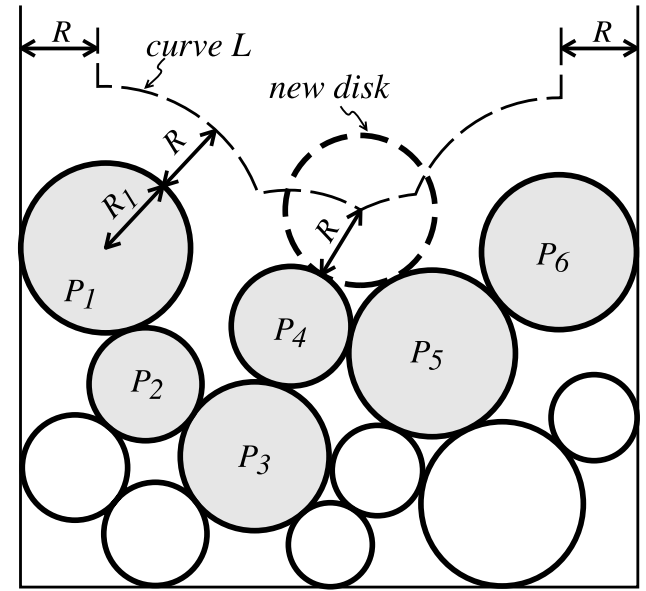

Figure 3. Construction of a numerical sample by means of geometrical rules. The center of each deposited disk is placed along the curve $L$ and at a point where the potential energy is minimum.

particle sets as, for example, dense samples of millimetric disks that preserve the relative size distribution and the texture.

[23] The particle size distribution is deficient in small sizes when compared with a natural well-graded soil, which may show a wider range of particle sizes $\left(R_{\mathrm{Max}} / R_{\mathrm{Min}}>100\right)$. Nevertheless, a range of the order of $R_{\mathrm{Max}} / R_{\mathrm{Min}}=4$ seems sufficiently wide to allow for a disordered two-dimensional configuration devoid of long-range (crystal-like) ordering. In soil mechanics, the uniformity of a sample composed of three-dimensional particles (e.g., spheres) is characterized by the uniformity coefficient $C_{u} \equiv D_{60} / D_{10}$ where $D_{10}$ is the grain size diameter corresponding to $10 \%$ finer and $D_{60}$ is the grain size corresponding to $60 \%$ finer [e.g., Coduto, 1999]. Applying this expression to our 2D particle sample gives $C_{u}=2.8 / 1.3=2.15$. Note that in $2 \mathrm{D}, C_{u}$ is related to the variation of area susceptible of exerting contact forces in the particle set, since the perimeter of the disks is proportional to the diameter.

\subsection{Geometrical Preparation}

[24] The particles are inserted one after another into a rectangular box. The radius of each new particle is generated according to a random process meant to produce a uniform distribution by weight. The position of each new particle is chosen to minimize an "energy function" $U$ in the permissible space (i.e., with no overlap with the already deposited particles). The energy function may be defined in several ways depending on the boundary conditions. The simplest situation arises when the force on a particle of mass $m$ is due to a uniform gravitational field $-m g \hat{z}$, where $g$ is the acceleration of gravity and $\hat{z}$ is a unit vector pointing upward. In this case, the energy function $U=m g z$ is simply proportional to the height $z$ of the particle. Hence the particle is placed such that the $z$ coordinate of its center is minimum in the permissible space. The energy function may also decrease near the two lateral vertical walls in order to ensure a more homogeneous distribution of the disks close to the geometric boundaries of the particle set.

[25] The numerical algorithm that is used to build the particle set is illustrated in Figure 3 . Let $\left[P_{1}, . ., P_{n}\right]$ be the chain of particles located on the top of the particle set and let $R$ be the radius of the new disk to be placed. Let $L$ be the curve located at a minimum distance $R$ to the disks $\left[P_{1}, .\right.$. , $\left.P_{n}\right]$ in the chain. The center of the new particle is located along curve $L$ at the point where the potential energy is minimum. This point will always correspond to the intersection between two (or possibly more) circle arcs. In practice, the position of each new particle according to these purely geometrical rules will always have at least two contact points with particles already deposited. In this way, the coordination number of the packing generated by this procedure is about four. Nevertheless, the coordination number decreases below four as soon as the true dynamics is applied. The packing has initially a volume fraction $\nu=$ 0.84 . Because of a wide polydispersity of our samples, this volume fraction is larger than that of a random close packing composed of monodisperse disks where $\nu=0.82$ [Troadec and Dodds, 1993].

\subsection{Uniaxial Compaction}

[26] The sample prepared by means of geometrical rules is compacted in order to get a dense packing of rectangular shape. The coefficient of friction between disks during compaction is set to zero in order to facilitate particle rearrangements. A normal stress $\sigma_{c}$ is applied on the right wall while other walls are fixed (Figure 1b). In these conditions, the reorganization of particles is random and the average distribution of contact orientations is quite homogeneous for all directions in the plane (with variations of less than $10 \%$ from the mean). The aspect ratio at the end of compaction is $h / l \approx 2.5$, where $h$ and $l$ are the height and the width of the sample, respectively.

\subsection{Application of the Confining Pressure}

[27] Before starting properly the biaxial compression test, the two vertical walls are removed and the confining pressure is applied directly on the disks. To do so, we select the particles located inside two vertical strips that are adjacent to the boundaries of the sample (Figure 1c). These layers of particles will be subjected to horizontal external forces applied at the center of mass of the disks. The thickness of the vertical strips is $7.5 \mathrm{~m}$, i.e., 2.5 times the mean diameter of the disks. The horizontal force on each disk is recalculated at each time step in order to keep the stress constant on the vertical strips. We will refer to this type of test as Deformable Wall (DW) test since the confining pressure is applied through deformable walls, materialized here by the particles in the strips.

[28] In order to prevent buckling or crushing of the sample against the horizontal walls during shear, a layer of particles is fixed to the top and bottom walls. These "bumpy" walls reduce at the same time the "layering effect" often observed in the vicinity of the walls. These boundary conditions ensure that the shear bands span the sample across the deformable walls.

\subsection{Biaxial Compression}

[29] During biaxial compression, the axial displacement of the top horizontal wall is increased at a fixed rate, the bottom horizontal wall remaining fixed (displacementcontrolled loading). The samples are shortened up to $10 \%$ of their initial height. The vertical strain is given 
by $\epsilon_{z}=\Delta z / h_{0}$, where $\Delta z$ is the axial cumulative displacement of the top horizontal wall (positive in the direction of compression), and $h_{0}$ is the initial height of the sample. The axial load is simply the sum of normal forces exerted by the disks on the top horizontal wall.

[30] Since we are interested in the quasi-static regime, the shear rate should be chosen so that the kinetic energy supplied by shearing is negligible compared to the work done by internal stresses per unit time and unit mass. This can be formulated in terms of an "inertia parameter" $I$ defined as

$$
I=\dot{\gamma} \sqrt{\frac{m}{p}}
$$

where $\dot{\gamma}$ is the shear rate, $m$ is the mean particle mass, and $p$ is the mean stress. The quasi-static limit is then characterized by the condition $I \ll 1$. The objective value of $\dot{\gamma}$ in the presence of shear bands is $\dot{\gamma} \approx V_{s p} / w_{s b}$, where $V_{s p}$ is the shortening displacement rate during the shear phase and $w_{s b}$ is the mean width of the active shear bands. In our simulations we find that $w_{s b}$ is roughly 5 times the mean disk diameter; see below. With $V_{s p}=0.5 \mathrm{~m} / \mathrm{s}$, we get $I \in$ $[0.007,0.002]$, which is an acceptable range, lower values of $I$ requiring longer simulations.

[31] A total number of 70 tests were done. The numerical simulations were performed by a personal computer with two processors of $2.4 \mathrm{GHz}$. The execution time for running five simultaneous high quality simulations is roughly 10 hours. The disk-disk coefficient of friction $\mu_{d d}$ was varied from 0.01 to 12 . Each sample (characterized by a friction coefficient $\mu_{d d}$ ), was tested for different values of the confining pressure $\sigma_{3}$, ranging from 0.5 to $8 \mathrm{MPa}$.

\section{Main Results}

\subsection{Stress-Strain and Volume Change Behavior}

[32] We first focus on the stress-strain behavior for a low and a high coefficient of friction, $\mu_{d d}=0.3$ and $\mu_{d d}=3$, respectively. These tests will be labeled DWL and DWH, where $\mathrm{L}$ and $\mathrm{H}$ stand for low and high friction, respectively.

[33] Figure 4a shows the axial stress $\sigma_{1}$ as a function of the axial strain $\epsilon_{z}$ for DWL (in gray) and DWH (in black) tests at $\sigma_{3}=8 \mathrm{MPa}$. The axial stress increases rapidly with strain and passes by a peak before decreasing to a plastic plateau. It shows low-amplitude (5\% of the mean stress) fluctuations which may be interpreted in terms of the propagation of instabilities, as explained below. Similar fluctuations have been observed in experiments with twodimensional particle sets [Ngadi and Rajchenbach, 1998]. It is suggested that stress pulses are generated by nucleation and propagation of transient cracks within packings of low friction monodisperse beads.

[34] The overall mechanical behavior corresponds to a hardening-softening rigid-plastic behavior as a characteristic feature of dense granular media composed of rigid particles [Roux and Radjai, 1998]. The hardening and softening aspects appear in the transient regime from the beginning of the test to the plastic plateau reached at $\epsilon_{z} \approx 0.05$. The mean peak strength $\bar{\sigma}_{\max }$ is defined as the maximum value of the mean vertical stress, for a mobile interval of $\Delta \epsilon_{z}=$ 0.002 along the strain axis. The residual strength $\bar{\sigma}_{\text {res }}$ is defined as the mean axial stress for $\epsilon_{z} \in[0.05,0.1]$ corresponding to the mean axial stress sustainable at large strains once the sample has failed.

\subsubsection{Low Friction}

[35] The curve of $\sigma_{1}^{\mathrm{DWL}}$ first shows a peak at $\epsilon_{z} \cong 0.002$, with $\sigma_{1}^{\mathrm{DWL}}=15 \mathrm{MPa}$. Then, a rapid decrease occurs, reducing $\sigma_{1}^{\mathrm{DWL}}$ down to $10 \mathrm{MPa}$ before increasing slowly again to $\sim 13 \mathrm{MPa}$ with a well-defined plateau reached at $\epsilon_{z} \approx$ 0.02 . The shape of the curve around the plateau is concave down. Beyond this plateau, $\sigma_{1}^{\mathrm{DWL}}$ gently decreases to $\sim 11 \mathrm{MPa}$ at $\epsilon_{z} \approx 0.04$. From this point onward, $\sigma_{1}^{\mathrm{DWL}}$ shows stress fluctuations with a wavelength $\sim \Delta \epsilon_{z} \in$ $[0.01,0.025]$ around a roughly constant mean value. The amplitude of stress fluctuations between consecutive extrema is about 1-4 MPa. Besides long-wavelength fluctuations, we observe small-amplitude fluctuations with a much shorter wavelength, as well as sharp peaks whose frequency increases with axial strain.

[36] The mean peak and residual strengths are $\bar{\sigma}_{\max }^{\mathrm{DWL}}=$ 14.7 $\mathrm{MPa}$ and $\bar{\sigma}_{\text {res }}^{\mathrm{DWL}}=12 \mathrm{MPa}$. Note that the main peak corresponding to $\bar{\sigma}_{\max }^{\mathrm{DWL}}$ is observed at the very beginning of the test where both the volume fraction and the coordination number are higher.

[37] The volumetric strain $\Delta V_{s}^{\mathrm{DWL}}$ during shear is also displayed in Figure 4a. The material is globally dilatant, yet very small contractional events can be observed. These events occur less frequently at the beginning than later. The mean dilation rate varies with the axial strain: For $\epsilon_{z}<$ $0.02, \Delta V_{s}^{\mathrm{DWL}}$ increases steadily at a mean rate of $\sim 0.4$, whereas for $\epsilon_{z} \in[0.02,0.04]$ the mean dilation rate decreases to $\sim 0.1$ and remains roughly constant for $\epsilon_{z}>0.04$. At the beginning of the test, the dilation occurs homogeneously in the whole sample. However, along with shear localization, the dilation is more and more localized within the shear bands [e.g., Desrues et al., 1996].

[38] The observed stress-strain and volume change behaviors are consistent with the well-known features of dense granular materials exhibiting peak and residual strengths and dilatant behavior. The initial state of the sample may be qualified as overcompacted. For loose samples, the initial peak would probably not be observed, whereas the residual stress would remain the same.

[39] Figure $4 \mathrm{~b}$ shows the number of rolling and slipping contacts, referred to as RCL and SCL, respectively, during the test for the low friction sample. At rolling contacts the friction force $T$ is below the Coulomb threshold $\mu N$, whereas slipping contacts imply that the Coulomb threshold is reached. RCL decreases from $\sim 6900$ to $\sim 6300$, and its mean value remains constant for $\epsilon_{z}>0.01$. SCL has a similar behavior, showing an initial decrease from $\sim 1600$ to $\sim 840$, followed by a roughly constant value. Note that RCL varies in phase opposition with SCL in the fully plastic state (the stress plateau) where the total number of contacts $\mathrm{NCL}=\mathrm{RCL}+\mathrm{SCL}$ remains constant on average.

[40] Figure 5 shows the evolution of NCL and the total number ZCL of contacts where $N=0$ (geometrical contacts with zero force). NCL decreases from $\sim 8500$ to $\sim 7100$ and ZCL decreases from $\sim 360$ to $\sim 160$. The coordination number $\bar{N} \equiv 2 \mathrm{NCL} / N^{\prime}$, where $N^{\prime}$ is the number of particles with two or more contacts, is $\bar{N}=3.9$ at the beginning of the shear phase, and it falls down to $\bar{N}=3.39$ at large strains. On the other hand, the number of isolated particles (i.e., with less than two contacts) increases from $7 \%$ at the 


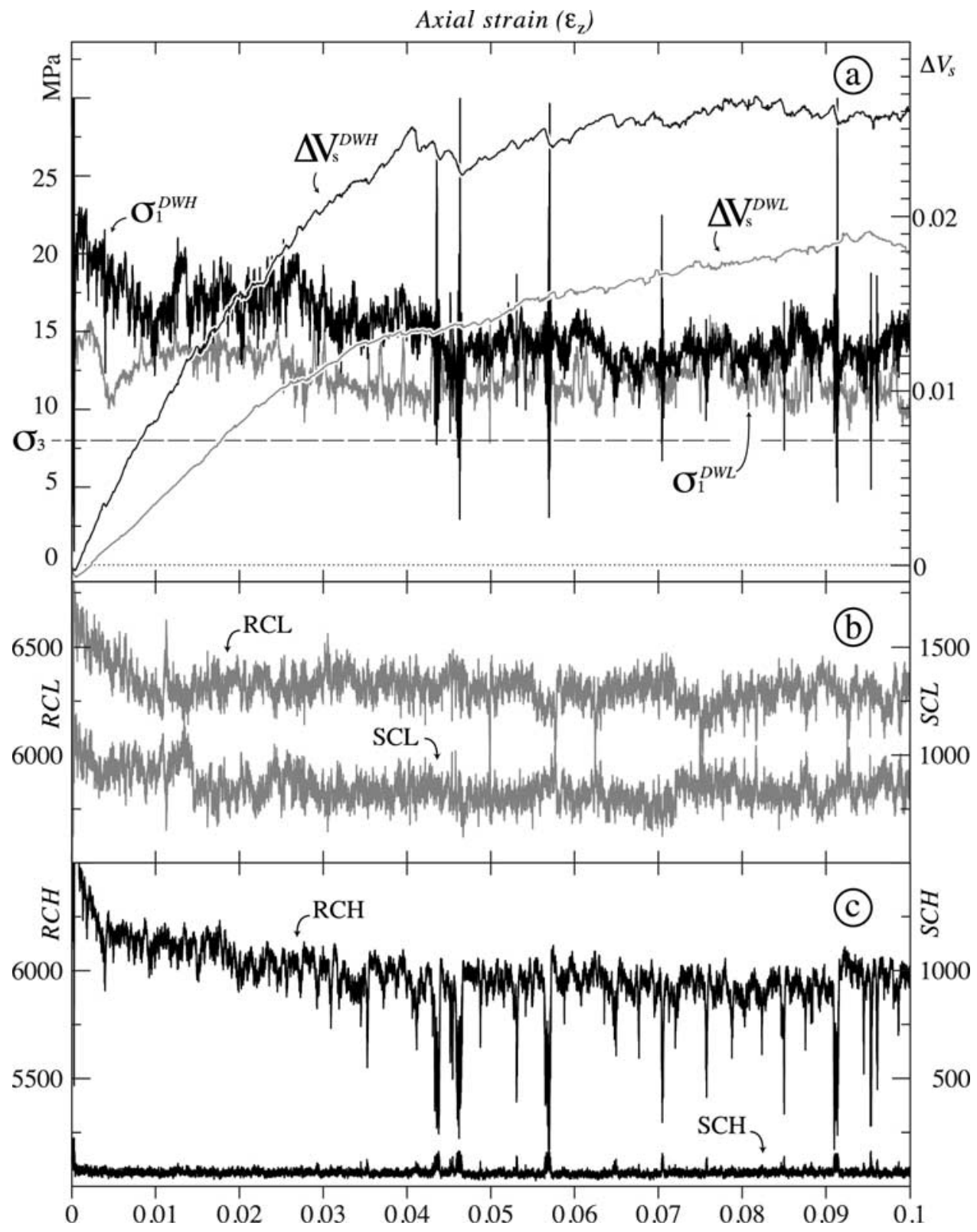

Figure 4. (a) Stress-strain and volumetric strain curves obtained for DWL and DWH biaxial tests for low (gray) and high (black) contact friction. Number of rolling and slipping contacts between the disks during shear for (b) low friction and (c) high friction.

beginning of the test to $15 \%$ at large strains. Note that the amplitude of short wavelength fluctuations of NCL and ZCL is much smaller than that of RCL and SCL. The coordination number in the fully plastic regime increases slightly with the confining pressure: Doubling $\sigma_{3}$, increases $\bar{N}$ by roughly $1 \%$. This is basically related to a slightly larger overlap between the particles at larger pressures. The mechanical behavior is scarcely modified by this slight change in coordination number.

[41] The correlation between the variations of the axial stress and the number of contacts can be observed in Figure 6a. which shows a zoom on the interval $\epsilon_{z} \in$ [0.047, 0.051]. We observe the following:

[42] 1. The axial stress shows small oscillations around successive plateaus, in which the sample dilates at a nearly constant rate and the number of contacts remains constant.

[43] 2. Stress plateaus are followed by a decrease in the axial stress down to a local minimum located at $1-4 \mathrm{MPa}$ beneath the mean stress at the previous plateau. The initiation of strain softening is indicated by means of vertical gray bands in Figure 6a. This stress fall is concomitant with a small contraction of the sample at an increasing rate. The total contraction during this phase is comparable with the volume of one average size particle. The stress minima are followed by one or several short-wavelength oscillations. The stress fluctuations at $\epsilon_{z} \approx 0.05$ are also clearly correlated with variations in the number of contacts: The number of slipping and zero force contacts increases sharply. All these observations may be interpreted in terms 


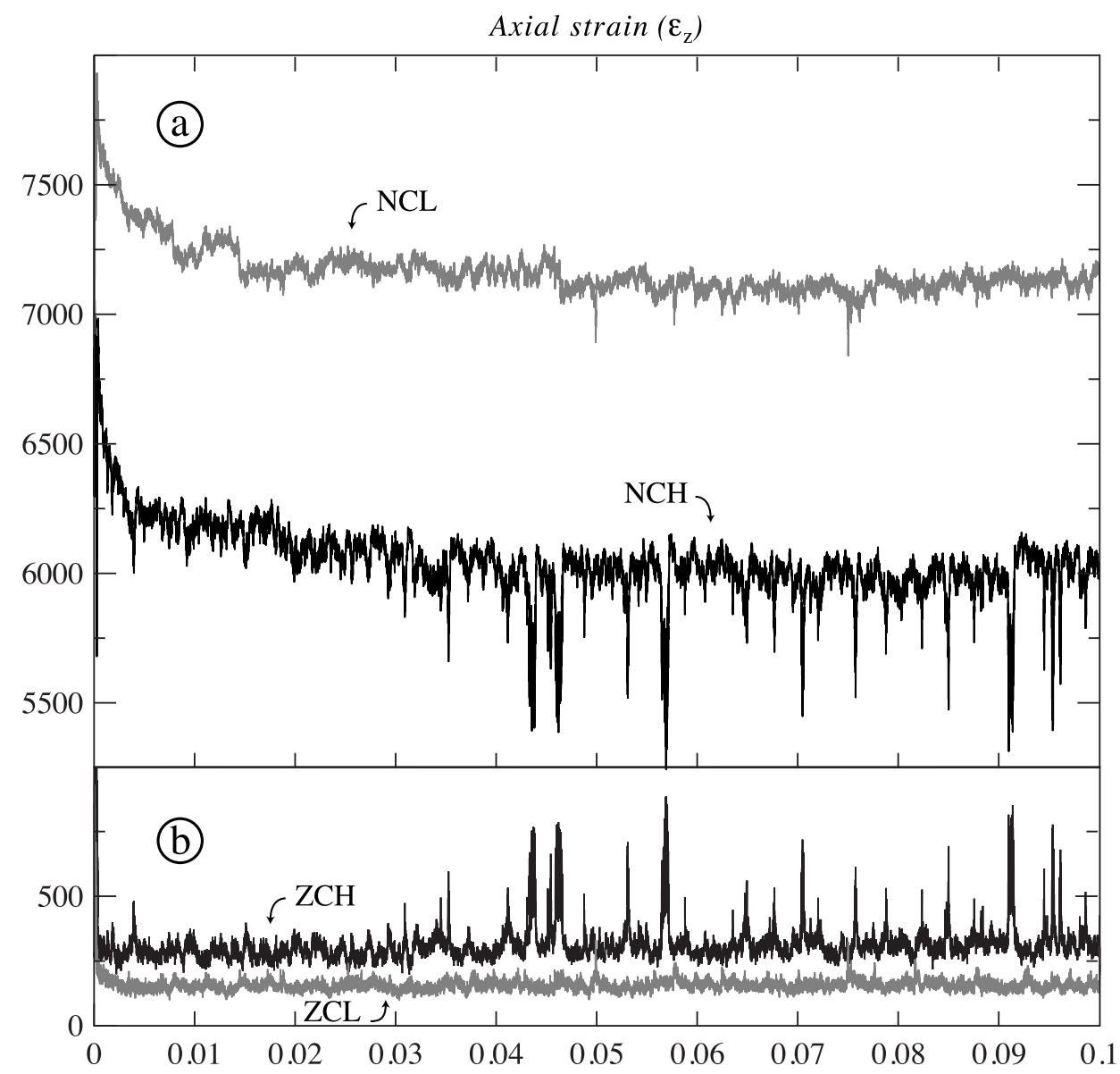

Figure 5. Number of contacts (a) with a nonzero force and (b) with zero force during shear for low (gray) and high (black) contact friction.

of the propagation of a dynamic shear instability within the sample during compression. Note that the volumetric strain rate becomes largely negative at this point indicating a small quasi-instantaneous contraction.

[44] 3. After the instability, the system tends to recover a new quasi-static state. The number of slipping contacts is lower than its value before the instability. The axial stress increases rapidly before falling gently to a new plateau (these deviations correspond to the sharp peaks described in Figure 4a).

\subsubsection{High Friction}

[45] The evolution of $\sigma_{1}^{\mathrm{DWH}}$ shows a well-marked peak at $\epsilon_{z} \approx 0.0015$, and then it decreases slowly exhibiting fluctuations around the mean value (Figure 4a). The mean peak strength is $\bar{\sigma}_{\max }^{\mathrm{DWH}} \cong 20.6 \mathrm{MPa}$. A minimum strength $\simeq$ $13 \mathrm{MPa}$ is reached at $\epsilon_{z} \approx 0.045$ followed by fluctuations around a constant value. The mean residual strength is $\bar{\sigma}_{\text {res }}^{\mathrm{DWH}}=13.7 \mathrm{MPa}$. The axial stress is globally higher for high friction than for low friction.

[46] The volumetric strain $\Delta V_{s}^{\mathrm{DWH}}$ during shear is also globally dilatant, yet small contractional events are observed (Figure 4a). For $\epsilon_{z}<0.02, \Delta V_{s}^{\mathrm{DWH}}$ increases at a mean rate of $\sim 0.8$. Thereafter, the mean dilation rate falls progressively to zero. The total dilation of the sample is greater for high friction than for low friction at any axial strain. The total number of contacts $\mathrm{NCH}$ is slightly above 6000 (Figures $4 \mathrm{c}$ and $5 \mathrm{a}$ ), and $\mathrm{SCH} \sim 60$ is one order of magnitude lower for high than for low friction. Thus, at high friction the particles tend to rearrange into a looser packing with higher strength and lower number of contacts. In the same way, $\mathrm{ZCH}$ is much larger for high friction than for low friction (Figure $5 b$ ).

[47] The mean coordination number for the high friction sample at the beginning of the test is $\bar{N}=3.9$. This value is the same as in the case of low friction. It decreases, however, to $\bar{N}=3.09$ at large strains. This is smaller than that for low friction. The number of isolated particles (i.e., with less than two contacts) increases from $7 \%$ at the initial stages of compression, to $20 \%$ at large strains.

[48] The evolution of $\sigma_{1}^{\mathrm{DWH}}$ is characterized by gentle increments followed by rapid falls. These fluctuations are observed all along the curve, and they are well correlated with volume changes and variations in the number of contacts as explained below. The mechanical behavior of the sample in the vicinity of one of these stress perturbations is shown in Figure 6b. We observe the following:

[49] 1. The variable $\sigma_{1}^{\text {DWH }}$ gently increases over a small strain interval $\Delta \epsilon_{z}$ and reaches a local maximum $\left(\Delta \epsilon_{z}\right.$ may vary between 0.0005 and 0.005$)$. The axial stress shows minor fluctuations around a concave-down mean curve. These fluctuations (observed both for low and high friction), reflect particle rearrangements as a distinctive feature of a nonsmooth mechanical processes. The vertical shortening $\Delta \epsilon_{z}$ is between 0.1 and $1 \mathrm{~m}$, which is slightly 

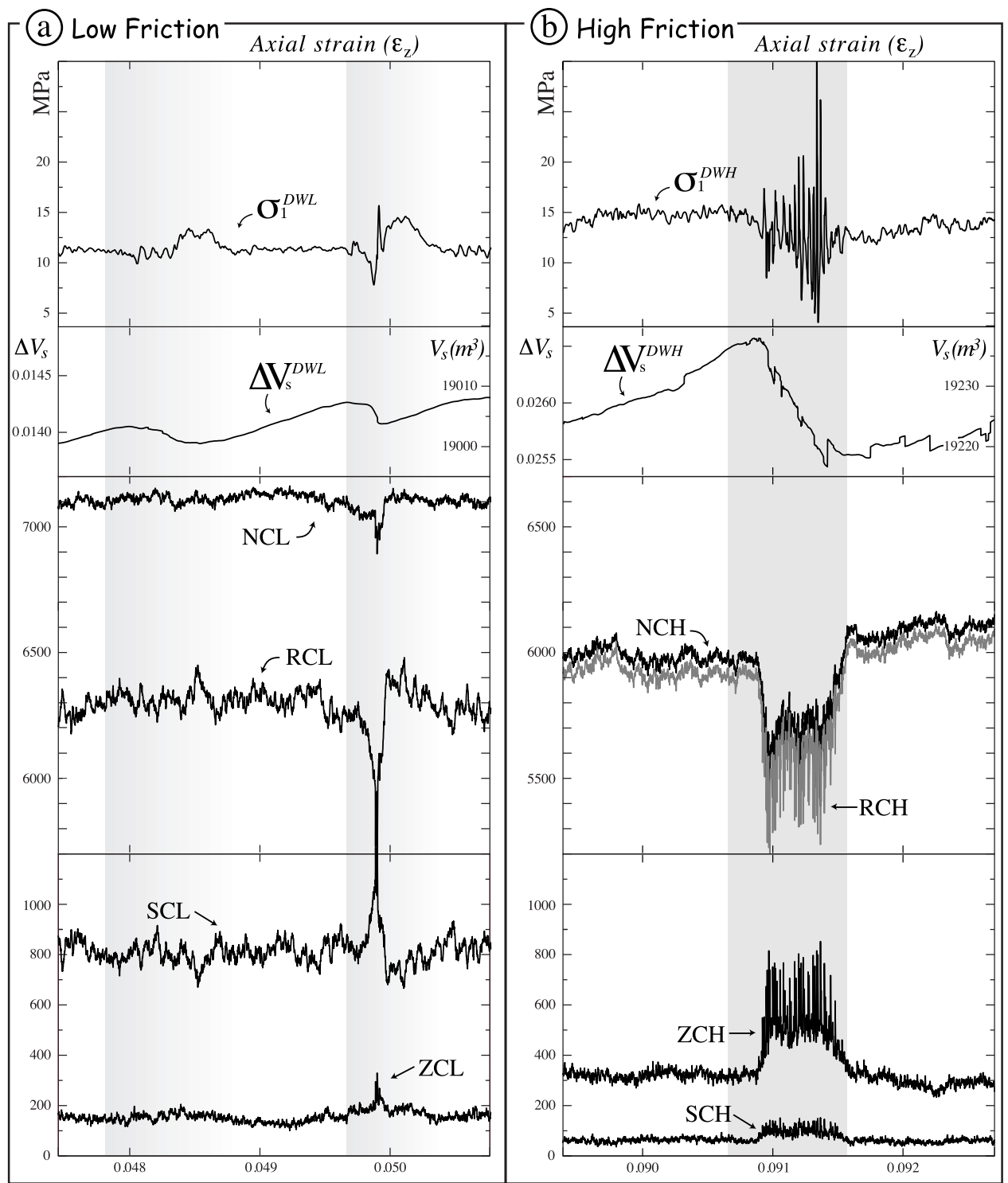

Figure 6. Axial stress, volumetric strain, and number of contacts as a function of axial strain in the vicinity of a shear instability for (a) low friction and (b) high friction.

below the mean particle radius. Simultaneously, the volumetric strain increases at a roughly constant rate and the number of contacts remains constant.

[50] 2. Beyond this point, the sample enters into a subcritical state in which a shear instability is about to propagate: The mean axial stress decreases as the volumetric strain rate falls to zero. At the same time, the number of slipping contacts begins to increase. At some point, yielding occurs and the sample enters into a transient state characterized by (1) a succession of minima and maxima whose amplitudes may reach up to $50 \%$ of the mean axial stress; (2) a rapid fall in volumetric strain (contraction); (3) a sharp reduction of the number of rolling contacts $\mathrm{RCH}$ and a slight increase in the number of slipping contacts $\mathrm{SCH}$; and (4) a net decrease in the total number of contacts $\mathrm{NCH}$ and a sharp increase in the number of contacts with negligible contact forces $\mathrm{ZCH}$.

[51] All these observations suggest an unstable regime characterized by "dynamic" rearrangements and collisions between particles along the shear bands. The strain interval corresponding to the initiation and propagation of the shear instability is indicated by a gray vertical band in Figure $6 \mathrm{~b}$.

[52] 3. After this unstable phase, $\sigma_{1}^{\mathrm{DWH}}$ is by $1-5 \mathrm{MPa}$ below the previous local maximum. $\mathrm{SCH}$ and $\mathrm{ZCH}$ fall to their means values.

[53] The correlation between shear strength and volumetric strain is similar to that observed by Morgan [1999], where granular shear zones were simulated in a simple shear geometry. The mechanical behavior bears a close analogy with the stick-slip instability observed for solid friction [Scholz, 1990; Mair et al., 2002]. There is, however, a major difference: In our simulations, no elastic energy can be stored. Nevertheless, because of volume changes, we may attribute a "configurational energy" to the system given simply by $p V$, where $p$ is the average stress and $V$ is the volume. Contractional events correspond thus to a loss of potential energy. This energy is dissipated by friction and inelastic collisions in the sample. The contractional events 
are enhanced once the initial dense particle set has undergone some expansion. Large contractions correspond to large stress peaks. This effect is more pronounced at high friction for which the volume changes are larger.

\subsection{Strain Localization}

[54] In this section, we analyze the deformation modes at low and high friction. We focus on the particle displacement and rotation fields at the final stages of the test. We also consider normal and tangential forces. Figure 7 illustrates the rotation and velocity fields in biaxial tests DWL and DWH at $\epsilon_{z} \approx 0.09$. Animation files illustrating the kinematic and static states of the samples are also available as auxiliary material ${ }^{1}$.

[55] In both DWL and DWH tests, we observe conjugated shear bands across the whole sample. Strain localization is frequently observed in numerical tests [e.g., Bardet and Proubet, 1992]. Shear bands can be clearly identified on the image illustrating the total displacements of the particles with respect to their initial positions. The magnitude of individual displacements is given by the continuous color scale located on the bottom of the image. The displacements varies from 0 to $25 \mathrm{~m}$ (purple to orange on the color scale). The particle rotation also provides a good indicator of the local shear strain as illustrated in Figure 7 (middle). The rotations are given in radians according to the color scale located below the image. Positive rotation is counterclockwise.

[56] The orientation of our shear bands is consistent with that of typical shear zones observed in samples of granular soil subjected to biaxial and triaxial laboratory tests [e.g., Bardet, 1997; Alshibli and Sture, 2000]. The mean angle between the shear bands and the axial stress both at low and high friction varies between $30^{\circ}$ and $40^{\circ}$. Shear bands are slightly curved and they become steeper close to the deformable walls. Shortening of the samples leads to the formation of a bottom and a top wedge bounded by active shear bands, which may be compared to normal faults resulting from an extensional stress regime (with vertical compression and horizontal extension). The top and bottom wedges are weakly deformed, as shown by their relatively homogeneous color (orange and purple, respectively). The shear strain is strongly concentrated along the shear bands, and, to a lesser extent, within the green triangular zones that are progressively extruded along the deformable walls. The initiation of conjugated shear bands is observed at very low strains and roughly around the initial stress peak. Shear bands propagate from local damage zones (strain inhomogeneities) that coalesce progressively into macroscopic shear zones (see the animations available as auxiliary material). These results are similar to those obtained from biaxial (plane strain) experiments on sand, in which early shear instabilities or bifurcations are observed [Vardoulakis et al., 1978; Peric et al., 1992].

\subsubsection{Low Friction}

[57] For low friction, the width of the shear bands increases progressively, reaching $12-16 \mathrm{~m}$ at the end of shortening (Figure 7, top). This thickness is of the order of 4 to 6 average particle diameters. Note that shear band

${ }^{1}$ Auxiliary material is available at $\mathrm{ftp}: / / \mathrm{ftp}$.agu.org/apend/jb/ 2003JB002955. thickness in cohesive granular media should be smaller due to a reduced mobility of the particles. Shear bands show evidence of a higher strain toward the top of the sample, and they are composed of several narrow fault planes across the particle set. Shear displacements and rotating particles tend to be localized along these planes that seem to form an anastomosed network pattern.

[58] The dilation is mainly concentrated along the shear bands, where the pores are larger than the average pore size (see the displacement field animation for low friction). The particles belonging to the right-dipping shear band show a higher degree of rotation and shear displacement. The sense of particle rotations along shear bands follows mostly the shear direction (synthetic disks). The most rapidly rotating particles are organized in chain-like patterns that are parallel to the shear band [Kuhn and Bagi, 2002]. The size range of synthetic disks is quite heterogeneous, and it includes both big and small particles. Nevertheless, we observe a considerable number of particles with opposite rotation (antithetic disks). Antithetic disks are generally small and they are usually in contact with one or more synthetic disks. The particle rotation (positive or negative) increases as the particle size diminishes. The analysis of the velocity field for the low friction sample shows that several conjugated fault planes may be activated simultaneously. In addition, fault planes are quite persistent in time and space. Particle rotation and displacement gradients decrease rapidly away from the shear planes. In fact, low friction favors energy dissipation along slipping contacts that are adjacent to the active shear planes. This suggests that the mechanical coupling between the shear planes and the neighboring particles is low.

\subsubsection{High Friction}

[59] For high friction, the conjugated shear bands are much more diffuse than for low friction, and their average thickness at the end of shortening is of the order of 8 to 10 mean particle diameters (Figure 7, bottom). Shear bands show a higher strain level toward the bottom of the sample, and they are composed of shear planes affecting a few particle layers. Dilation occurs mainly along the shear bands. The green triangular zones adjacent to the deformable walls show a larger amount of porosity than in the low friction case. Particle rotations are quite comparable for high and low friction, yet some significant differences are observed: (1) small particles with large rotations (positive and negative) are many more at high friction; and (2) rotating small particles are located in the shear bands, and within the adjacent areas (in the triangular zones, and to a minor extent, in the top and bottom wedges). The velocity field illustrated in Figure 7 (bottom, right), was obtained during a dilatant period. The particle velocities are much more chaotic and diffuse than for low friction. Nevertheless, we still observe shear planes characterized by a discontinuous velocity field across the shear zone. Shearing may jump successively from one rupture plane to another over a small time interval. Particle rotations and displacement gradients are distributed within a larger volume around the shear planes.

\subsection{Contact Forces}

[60] The normal and tangential contact forces are illustrated as rectangles of different colors connecting particle centers (Figure 8). The width of each rectangle is propor- 


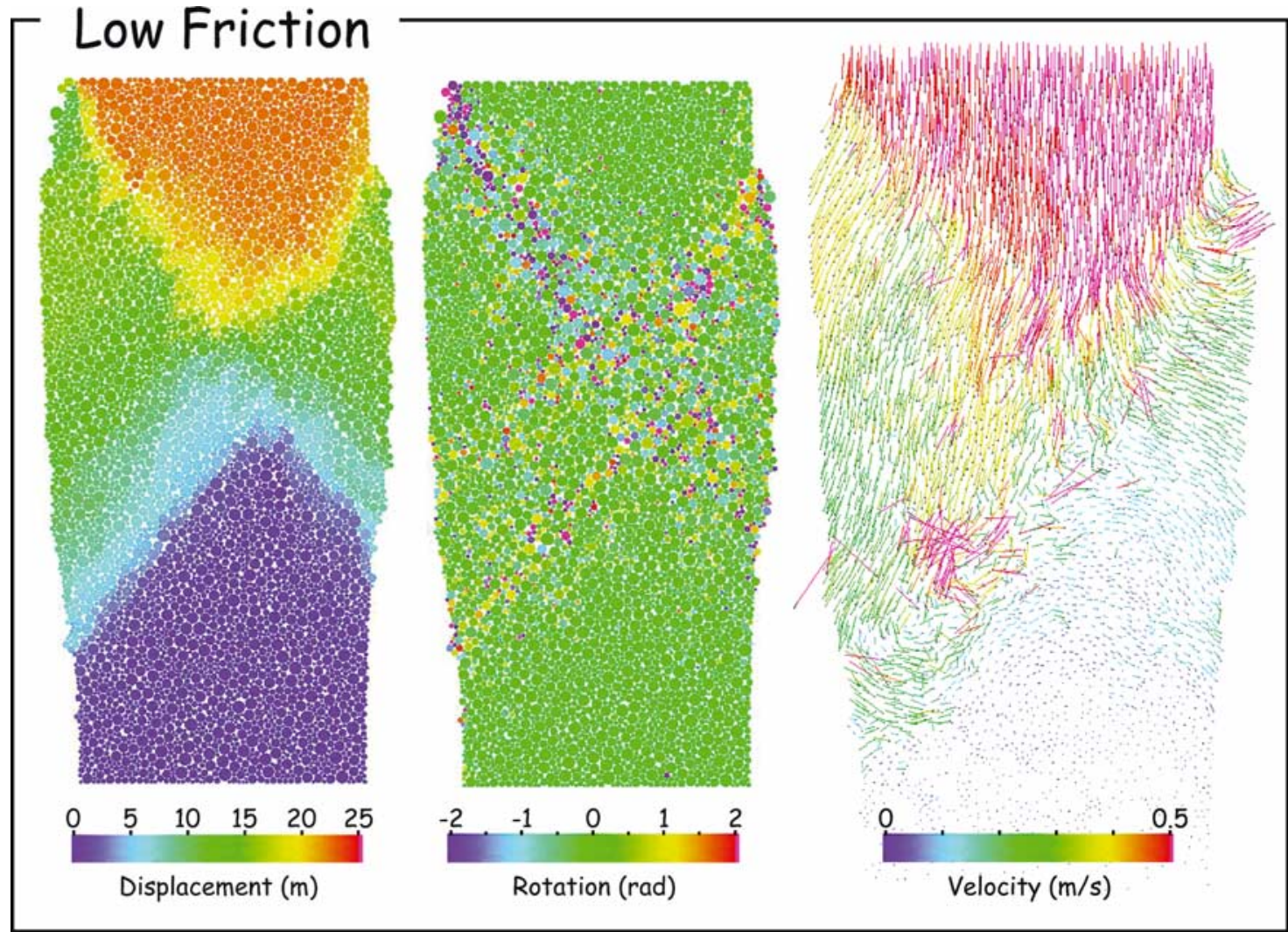

\section{High Friction}
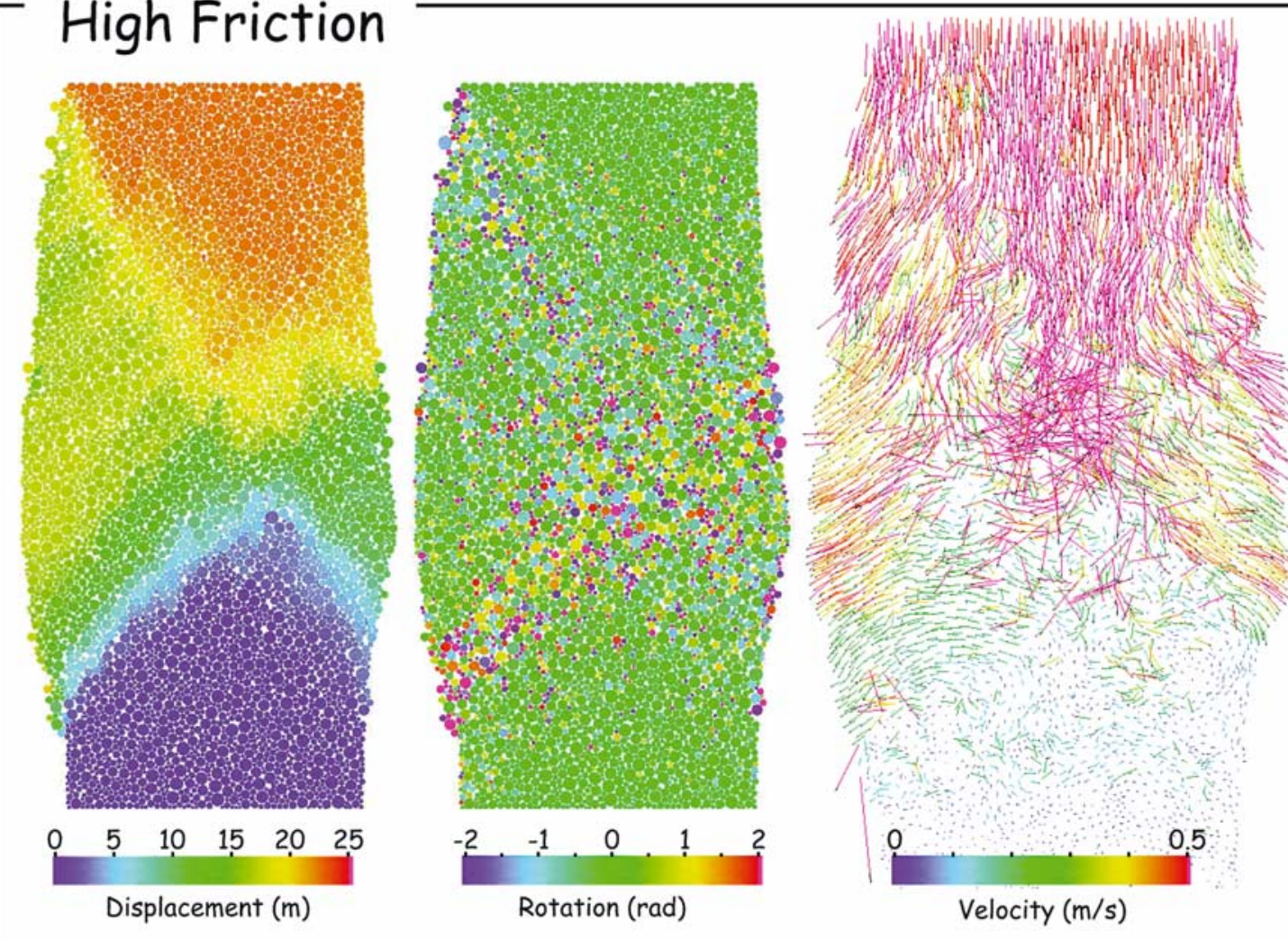

Figure 7. Total displacement (from the beginning of the test), rotation and velocity fields for low and high contact friction at $\epsilon_{z} \approx 0.09$. 


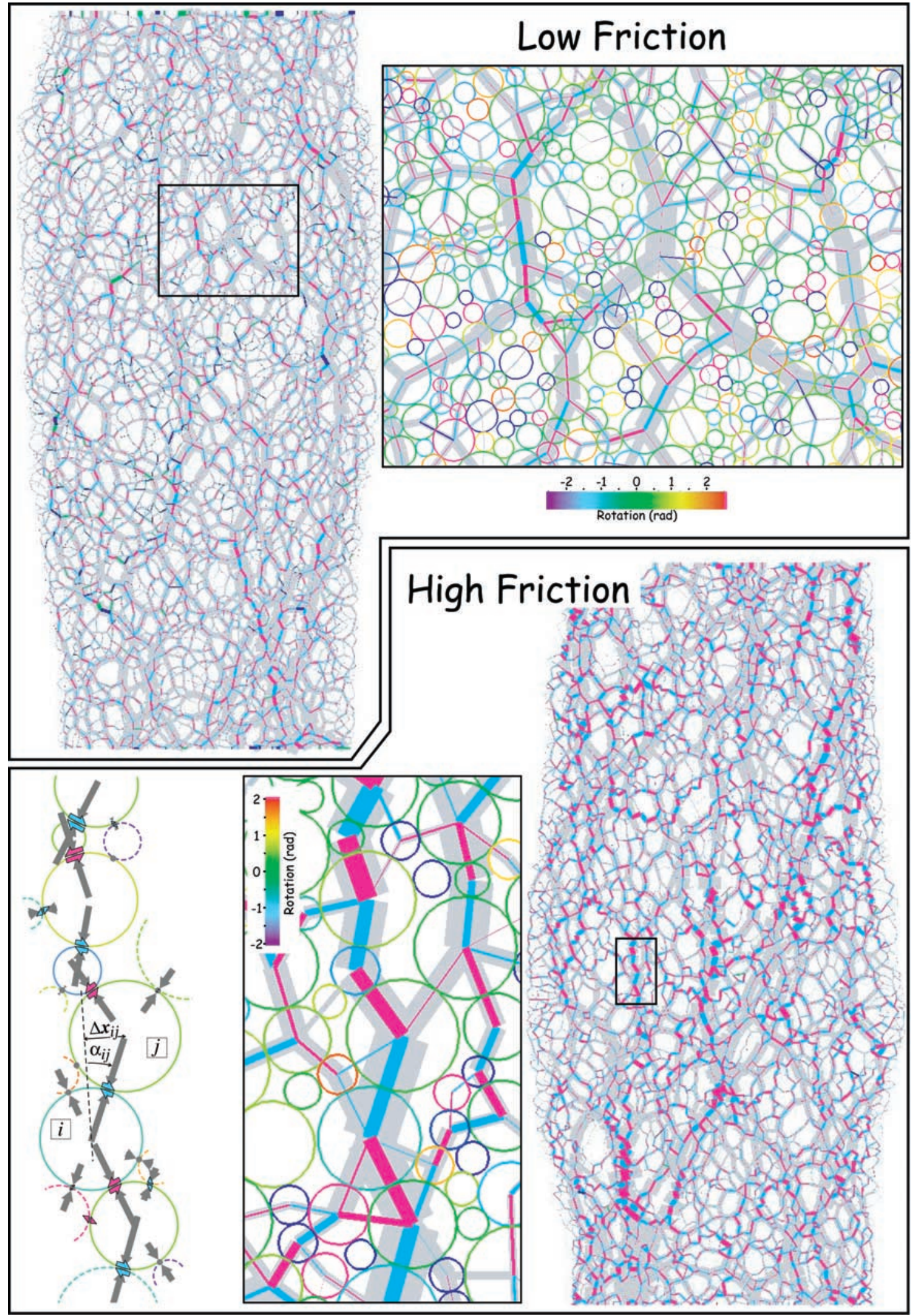

Figure 8. Contact forces for low and high friction biaxial tests, represented as rectangles of different colors. Detailed views of contact forces and particles are shown in blow-ups. The axis of each rectangle is the line connecting the disk centers; the width is proportional to the magnitude of the contact force; $\epsilon_{z} \approx$ 0.09; gray, normal force; pink and blue, positive and negative friction forces at rolling contacts; purple and green, positive and negative friction forces at sliding contacts. 
tional to the magnitude of the contact force. Normal forces $N$ are in light gray. Friction forces $T$ are represented by means of the following color code: The positive and negative forces below the Coulomb threshold are plotted in pink and blue, respectively, whereas the positive and negative forces at the Coulomb threshold are plotted in dark purple and green, respectively. Positive tangential forces induce counterclockwise torques on both particles.

[61] Both for low and high friction, the normal forces are characterized by chains of strong forces whose magnitudes are above the mean force [Radjaï et al., 1998]. These chains define a zigzag trajectory with particles located at the vertices. The chains of normal forces are, on average, parallel to the axial stress, and they are interconnected through bifurcations and arches. Nevertheless, stress deviations may be observed within the sample, in particular, close to the shear bands. Note that the sign of the friction force changes periodically (from pink to blue) along consecutive contacts in a chain.

[62] The stability of strong chains involves two factors (Figure 8): (1) friction forces that are exerted between contiguous particles in the chain and (2) lateral normal forces exerted by particles adjacent to the chain. Let $\alpha_{i j}$ be the angle between the normal contact force and the mean direction of the force chain (Figure 8, bottom). Concerning the first factor, the magnitude of the friction force increases when $\alpha_{i j}$ becomes greater. In this situation, the tangential component tends to reorient the total contact force parallel to the mean direction of the chain so as to ensure the equilibrium of the chain. $\alpha_{i j}$ can take higher values (up to $90^{\circ}$ ) for high friction than for low friction, the zigzag trajectory being more squeezed. These results may be illustrated by a simple experience: try holding a ball with two fingers. If the fingers are located at opposite points then the ball will be quite stable since low frictional forces are applied at the contact points. If you move your fingers close, then frictional forces become higher and with opposite sense, and the equilibrium is more difficult to sustain.

[63] The particles located in between the chains are organized in a weak network of contacts including forces whose magnitudes are less than the mean force. The weak network shows a greater number of interconnections for low than for high friction. In fact, particle chains are intrinsically less stable at low friction, and they require statistically more lateral supports to achieve equilibrium. These lateral supports usually correspond to adjacent particles from the weak network [Radjaï et al., 1998]. These observations are consistent with the volumetric strain curves in Figure 4a, which show that total dilation is greater for high than for low friction so that $\mathrm{NCH}<\mathrm{NCL}$.

[64] The configuration of strong and weak chains is correlated with the texture. As a general rule, force chains involve particles larger than the average (Figure 8). Indeed, the strong forces appear mostly along particle strings that are subparallel to the axial stress. This means that the particle strings composed of big disks are, on average, stronger and more stable than strings that include small disks. This behavior results from a very simple stability concept: When particles of different sizes are piled up, the packing is generally destabilized close to the smallest elements. Let $i$ and $j$ be two consecutive circular particles located along a chain (Figure 8, bottom). Let $\Delta x_{i j}$ be the distance between the disk centers perpendicularly to the axis of the chain $\left(\Delta x_{i j}>0\right.$ if the particles are not perfectly aligned with the chain's axis). For a given distance $\Delta x_{i j}$, the angle $\alpha_{i j}$ increases as the size of the particles decreases. Thus small particles usually require greater friction forces along the chain to achieve equilibrium, and they are more easily disturbed than larger disks.

[65] The small particles are occasionally observed along strong chains, especially during the early stages of the shearing. These particles become unstable (especially for low friction), and they are progressively expelled laterally. Unstable disks often induce bifurcations in the strong chains, as well as local rearrangements in the particle set. These observations suggest that local segregation processes related to the heterogeneous force distribution can operate during shear. Lateral expulsion of small particles may reduce readily the axial strength and thus initiate the propagation of shear instabilities across the sample. Lateral expulsion of particles located along a strong force chain has been proposed to explain stress fluctuations in experimental compression tests [Ngadi and Rajchenbach, 1998].

[66] The heterogeneous distribution of forces as a function of the particle size has important consequences on the deformation processes observed in natural examples. For instance, stress concentrations observed along contact chains may induce grain splitting of large particles deformed under high confining pressures. This process may lead to a balance between grain sliding and crushing as a mechanism for the generation of self-similar size distributions within shear zones [Biegel et al., 1989].

[67] The particle rotations are also correlated with the distribution of forces within the particle set (Figure 8). Major rotations involve principally the small particles that are adjacent to the chains of strong forces. Thus shear interactions may induce rotation of these particles within the expanded zone located near the chains. Mechanical coupling between rotating particles is currently observed in the weak network: Adjacent particles may rotate in opposite directions. In this sense, the weak network may be compared to a fluid-like medium where particle movement is less constrained, whereas particle chains resemble a solid-like medium that absorbs normal and shear stresses [Radjaï et al., 1999].

\subsection{Shear Instabilities}

[68] The zones of instability may be identified with the particles whose velocity is well above the shortening rate (Figure 9). For low friction, velocity instabilities are located along narrow bands close to the shear planes. For high friction, the instabilities appear along larger and thicker shear bands and they may propagate across the whole sample. The velocities of unstable particles follow a sort of fluctuating wavy pattern around the mean shear direction. These instabilities are systematically observed during the small contractional events described previously, and they may be compared to the velocity field observed around a fault plane during an earthquake. Velocity fluctuations are characteristic of nonsmooth mechanical processes (they do not correspond here to numerical noise) [Radjai and Roux, 2002]. Coseismic velocities and displacements are higher in the vicinity of the shear plane and they fall away from the 


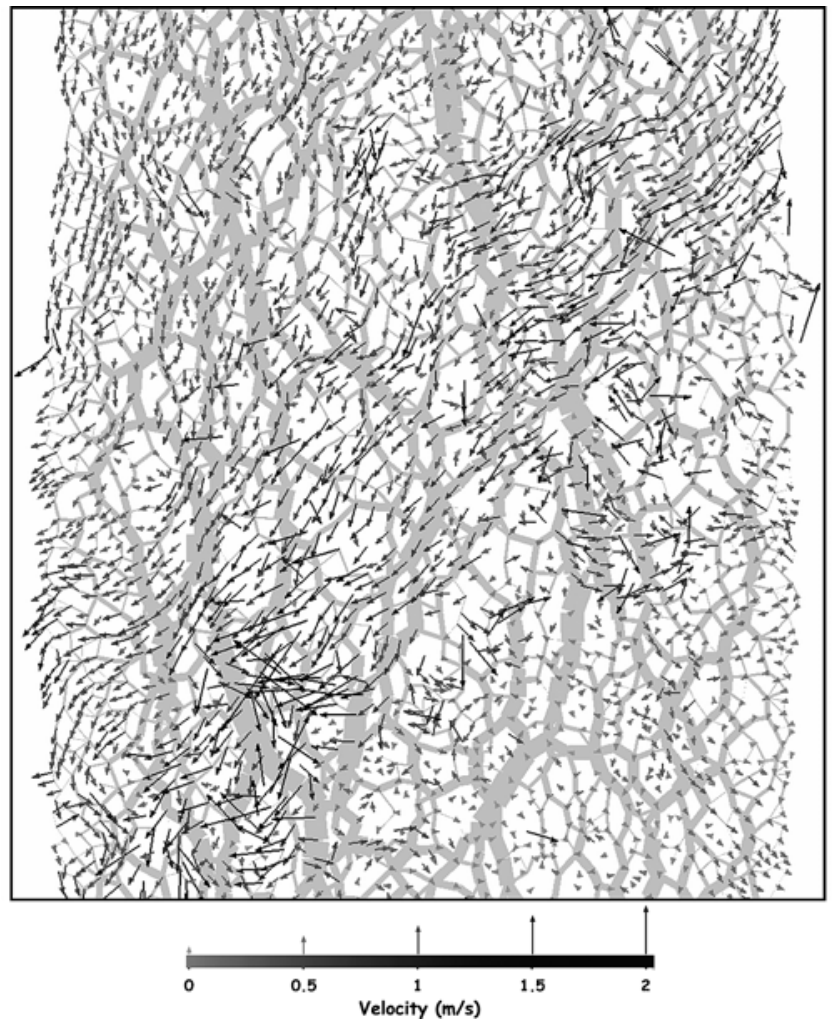

Figure 9. Velocity field of the disks and normal contact forces observed during the propagation of a shear instability within the sample for high contact friction. The shortening displacement rate $V_{s p}=0.5 \mathrm{~m} / \mathrm{s}$.

fault. These critical velocities underly the macroscopic stick-slip behavior discussed in section 4.3.

[69] As expected, stress deviations are observed close to the shear bands [Petit and Barquins, 1990; Baotang et al., 1995], both for low and high friction (Figure 9). The force chains are frequently deviated as they approach the shear planes, tending to become parallel or perpendicular to the rupture planes. Note that when the principal stress direction becomes parallel or perpendicular to a shear plane, the shear stress becomes minimal. This behavior suggests that the shear bands correspond to macroscopic zones of weakness with a lower shear strength than the bulk.

\subsection{Shear Strength Versus Contact Friction}

[70] In order to study the influence of contact friction on the shear strength, we analyzed the stress-strain behavior for the following set of friction coefficients:

$$
\mu_{d d} \in[0.01,0.1,0.2,0.3,0.4,0.5,0.6,0.8,1,1.2,3,6,12] .
$$

Each sample (characterized by a friction coefficient), was sheared for the following set of confining pressures (in $\mathrm{MPa})$ :

$$
\sigma_{3} \in[0.5,1,2,4,8] .
$$

For each test we determine both the maximum and residual strengths $\bar{\sigma}_{\max }$ and $\bar{\sigma}_{\text {res. }}$. For a given value of $\mu_{d d}$, the behavior is characterized by plotting the results of the tests in $p-q$ space for different confining pressures, where $p=$ $\left(\sigma_{1}+\sigma_{3}\right) / 2$ is the mean stress, and $q=\left(\sigma_{1}-\sigma_{3}\right) / 2$ is the stress deviator. We did not observe a systematic variation in the orientation of shear bands as a function of confining pressure. Figure 10a displays the peak failure envelope obtained from a linear regression, which fits quite well the five data points obtained for $\mu_{d d}=3$. The coordinates of each point are calculated by setting $\sigma_{1}=\bar{\sigma}_{\max }$. The fitting line passes through the origin implying that the cohesion intercept is zero. The regression line allows to calculate the classic Mohr-Coulomb failure line, which is tangent to the Mohr circles at rupture. The macroscopic shear strength may be characterized by the slope of the Mohr-Coulomb line, which is given by

$$
\begin{aligned}
\mu_{\text {macro }} & =\tan \phi \\
\sin \phi & =q / p .
\end{aligned}
$$

The residual strength envelope is calculated in the same way, by taking $\sigma_{1}=\bar{\sigma}_{\text {res }}$. The slope of the corresponding Mohr-Coulomb residual failure line is termed $\mu_{\text {macro }}^{\text {res }}$ We find that the cohesion intercept is zero for all values of the contact friction $\mu_{d d}$. Hence the macroscopic behavior is characterized by only two parameters: $\mu_{\text {macro }}^{\text {res }}$ and $\mu_{\text {macro }}$.

[71] Figure 10b shows the macroscopic friction parameters, $\mu_{\text {macro }}$ and $\mu_{\text {macro }}^{\text {res }}$ as a function of the contact friction coefficient $\mu_{d d}$. The macroscopic friction increases rapidly at low values of $\mu_{d d}$. In other words, a slight increase of contact friction in this limit has a considerable impact on the equilibrium states of the particle set. On the contrary, for
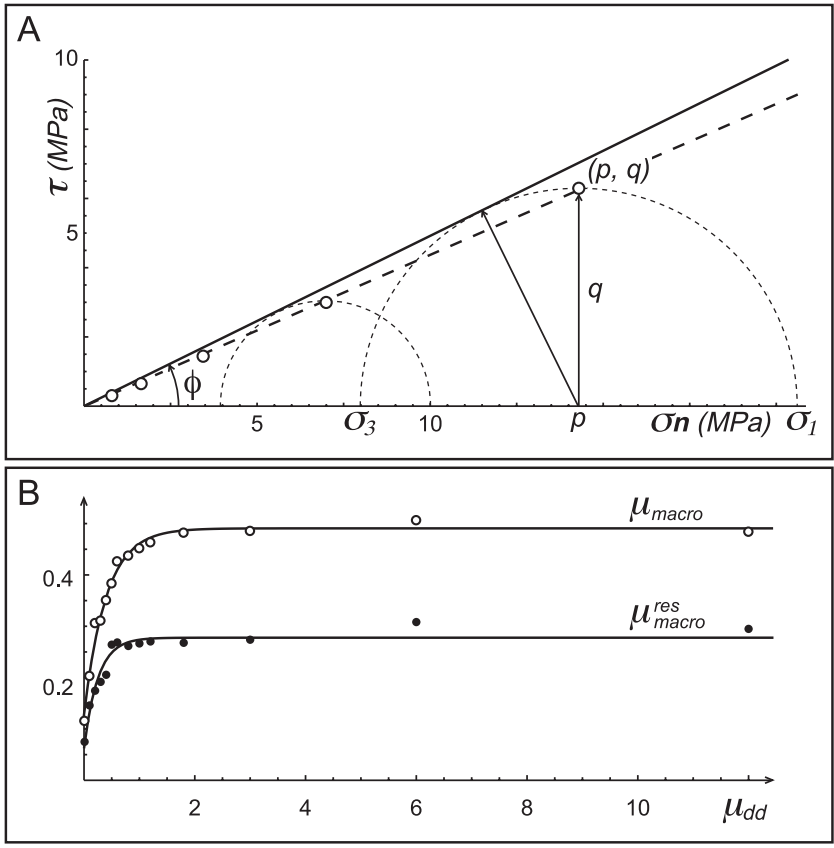

Figure 10. (a) Peak failure envelope obtained from a linear regression to fit the five data points obtained for $\mu_{d d}=$ 3. $p=\left(\sigma_{1}+\sigma_{3}\right) / 2$ and $q=\left(\sigma_{1}-\sigma_{3}\right) / 2$. (b) Relationship between the contact friction $\mu_{d d}$ and the macroscopic friction parameters $\mu_{\text {macro }}$ and $\mu_{\text {macro }}^{\text {res }}$. The data are fitted by means of concave down exponential functions. 
larger values of $\mu_{d d}$, both $\mu_{\text {macro }}$ (for $\mu_{d d}>2$ ) and $\mu_{\text {macro }}^{\text {res }}$ (for $\left.\mu_{d d}>1\right)$ reach an asymptotic value. These results are consistent with those obtained by means of usual discrete element methods [e.g., Bardet and Proubet, 1992; Oger et al., 1998].

[72] The data are fitted by means of a concave down exponential function:

$$
\begin{aligned}
& \mu_{\text {macro }}\left(\mu_{d d}\right)=0.49-0.37 e^{-2.5 \mu_{d d}} \\
& \mu_{\text {macro }}^{\text {res }}\left(\mu_{d d}\right)=0.28-0.22 e^{-4 \mu_{d d}} .
\end{aligned}
$$

The intercepts of the exponential functions with the vertical axis are given by $\mu_{\text {macro }}(0)=0.12$ and $\mu_{\text {macro }}^{\text {res }}(0)=0.06$. These offsets define the macroscopic friction parameters for frictionless particles. This means that the macroscopic shear strength persists even at zero contact friction, suggesting that inelastic collisions at the particle scale lead to Coulomb-like frictional behavior at the macroscopic scale [Roux and Radjaï, 2001].

[73] The global response obtained for circular particles, may be extended to other grain shapes such as polygons. Indeed, we expect that angularity just tends to strengthen the sample and slow down local or global phenomena such as shear band formation [Nouguier-Lehon et al., 2003]. Indeed, the macroscopic coefficient of friction is higher for angular grains than for disks. For three-dimensional particle sets, the shear strength is higher since there is a larger number of kinematical constraints in spite of the extra degrees of freedom (e.g., $\bar{N} \in[4.5,6]$ ) [Frye and Marone, 2002]. For instance, the angle of stability of a sandpile is higher than for a pile of disks. Nevertheless, additional simulations are required to determine quantitatively the influence of grain shape and angularity on the global behavior of granular materials.

[74] The analysis of biaxial tests shows that the shear strength of a shear band in a granular material results from two different mechanisms operating simultaneously at the particle scale: The first mechanism is simply friction mobilization along a space direction or along the shear band when the latter is present. The second mechanism is the roughness of the shear zone, which is intimately related with the particle size distribution and the texture of the sample. Particles along a shear band may be viewed as obstacles or asperities that restrain the relative displacement of the disks parallel to the shear zone. Imbricated particles subjected to shearing exert normal contact forces on each other that resists shear movement. The projection of compressive normal forces along the shear band largely contributes to the macroscopic shear strength. Thus before a shear instability propagates, particles must climb above obstacles, or displace the asperities along the shear zone. This "climbing process" generates expansion and corresponds to the quasi-static deformation phase described before. In principle, this mechanism may operate even if the friction coefficient at the contacts is zero. From an energetic point of view, this mechanism works if the collisions are inelastic and thus dissipate the accumulated potential energy.

[75] The stress deviations observed in the vicinity of shear bands are also related to this mechanism: If a chain of force is perpendicular to a shear band, then the contact planes between particles in the chain are subparallel to the shear direction. Thus the projection of contact normal forces along the shear plane is very low, and its contribution to the macroscopic shear strength is negligible.

\section{Conclusions}

[76] The contact dynamics method is suitable for the description of geological processes involving large plastic deformations of heterogeneous particle collections. The mechanical behavior of frictional granular materials was analyzed by means of biaxial numerical tests performed on samples composed of polydispersed disks, using the contact dynamics method. The main results of this study can be summarized as follows:

[77] 1. Perfectly rigid particle systems analyzed by means of the contact dynamics method have a macroscopic plastic behavior that is consistent with that observed for systems consisting of more or less deformable (elastoplastic) particle systems and simulated by usual discrete element methods. Nevertheless, stress fluctuations seem to be enhanced in the absence of contact deformability or damping effects. The mean mechanical behavior is a hardening-softening rigid-plastic behavior governed by a Mohr-Coulomb yield function.

[78] 2. Shearing results in the formation of conjugated shear bands across the system. The mean angle between the shear bands and the axial stress for low and high friction between the particles is the same and it varies between $30^{\circ}$ and $40^{\circ}$. Dilatancy occurs mainly within the shear bands. The width of the shear bands at $\epsilon_{z}=0.1$ increases from 5 to 10 average particle diameter as the contact friction increases. The particle rotations along shear bands mostly follow the shear direction, although we observe a considerable number of small particles rotating in the opposite direction. The mechanical coupling between the shear planes and the neighboring particles increases as the contact friction rises.

[79] 3. The axial stress increases rapidly with strain and passes by a peak before falling to a plastic plateau showing low-amplitude fluctuations.

[80] 4. The influence of contact friction $\mu_{d d}$ on macroscopic friction coefficients for peak and residual strengths ( $\mu_{\text {macro }}$ and $\left.\mu_{\text {macro }}^{\text {res }}\right)$ is analyzed via the construction of the Mohr-Coulomb envelopes. The macroscopic friction increases rapidly at low values of $\mu_{d d}$, whereas for larger values of $\mu_{d d}$, both $\mu_{\text {macro }}$ and $\mu_{\text {macro }}^{\text {res }}$ reach an asymptotic value. We find that the macroscopic shear strength persists even at zero contact friction, suggesting that inelastic collisions at the particle scale lead to Coulomb-like frictional behavior at the macroscopic scale.

[81] 5. The volumetric strain during the shear test is globally dilatant and localized within the shear bands. The total dilation is always larger for high friction than for low friction. The number of slipping contacts decreases with contact friction.

[82] 6. The fluctuations of the axial stress around the mean are characterized by gentle increments followed by rapid falls, in close analogy with the stick-slip behavior of solid friction. These stress falls are concomitant with small contractional events, suggesting the propagation of dynamic shear instabilities.

[83] 7. Strong force chains follow a zigzag trajectory with particles located at the vertices. These chains are, on 


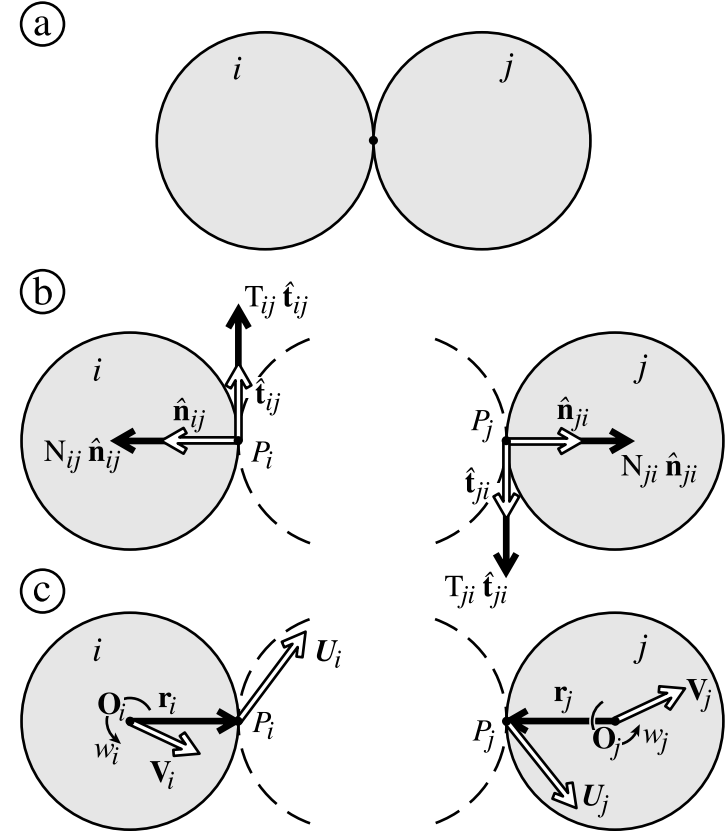

Figure A1. (a) Example of two arbitrary disks $i$ and $j$, which are in contact at a given point. (b) Normal and friction forces acting at the contact point, expressed for each particle in the local reference frame. (c) Velocities of the center of mass of each disk, and of the material points $P_{i}$ and $P_{j}$ coinciding with the contact point.

average, parallel to the axial stress, though deviations occur in the vicinity of the shear bands. The zigzag pattern is more pronounced at high friction. The sign of the friction force changes periodically along consecutive contacts in a chain. The chains are basically stabilized by weak forces from lateral supporting particles.

[84] 8. The force chains involve mostly particles larger than the average. Small particles are occasionally observed along strong chains. These particles become unstable and they are progressively expelled from the chain. Small particles in contact with strong chains exhibit large rotations

[85] Some of these microscopic features suggest plausible mechanisms that can be relevant in the geological context. In this respect, it is of interest to consider the application of the contact dynamics approach to simulate heterogeneous geological structures or dynamic processes involving tectonic deformations and gravitational phenomena. However, such applications imply the use of more complex interactions at the particle scale. In particular, cohesion and aging phenomena are certainly of primary importance at geological timescales. From a purely numerical standpoint, the contact dynamics method provides an attractive alternative to the usual discrete element methods used in geosciences. The present contribution in a geological background is an attempt in this direction.

\section{Appendix A: Contact Dynamics Algorithm}

[86] In this appendix we detail the algorithmic features of the contact dynamics (CD) method as a distinct element numerical approach. We present an "operational" descrip- tion of this method in view of its implementation in a numerical code.

[87] Let us consider two disks $i$ and $j$, located close to each other (Figure A1a). If the disks are in contact, they will exert forces on each other at their contact point. Let $P_{i}$ and $P_{j}$ be the material points belonging to the boundaries of the disks and coinciding with the contact point. In order to describe the forces and velocities at the contact point, we define a local reference frame given by two orthonormal vectors $\hat{\mathbf{t}}_{i j}$ and $\hat{\mathbf{n}}_{i j}$, which are tangent and normal to the contact surface, respectively (Figure A1b). The reference frame $\left(\hat{\mathbf{t}}_{i j}, \hat{\mathbf{n}}_{i j}\right)$ is linked to particle $i$, and it will be used to describe the forces applied by particle $j$ on particle $i$. Note that $\hat{\mathbf{t}}_{i j}$ and $\hat{\mathbf{n}}_{i j}$ are parallel and perpendicular to the common tangent plane.

[88] Let $T_{i j} \hat{\mathbf{t}}_{i j}$ and $N_{i j} \hat{\mathbf{n}}_{i j}$ be the shear and normal forces exerted by particle $j$ on particle $i$ at the contact point. By convention, we set $N_{i j} \geq 0$ when the normal contact force is compressive. Repulsion between particles is necessary to prevent interpenetration once the contact has been established. The normal force cannot be negative unless the contact is cohesive and it can support traction.

[89] Symmetrically, we define a local reference frame $\hat{\mathbf{t}}_{j i}$ and $\hat{\mathbf{n}}_{j i}$ linked to particle $j$, that will be used to describe the forces exerted by disk $i$ on disk $j$ (Figure A1b). The shear and normal forces acting on particle $j$ are given by $T_{j i} \hat{\mathbf{t}}_{j i}$ and $N_{j i} \hat{\mathbf{n}}_{j i}$, where $T_{j i}=T_{i j}, N_{j i}=N_{i j}, \hat{\mathbf{t}}_{j i}=-\hat{\mathbf{t}}_{i j}$ and $\hat{\mathbf{n}}_{j i}=-\hat{\mathbf{n}}_{i j}$. These relationships satisfy Newton's third law (action and reaction). In this representation, the contact forces are described by two unique scalar values $\left(T_{i j}, N_{i j}\right)$.

\section{A1. Local Kinematics}

[90] The velocity $\mathbf{U}_{i}$ of the material point $P_{i}$ located in the perimeter of disk $i$ can be expressed in the local reference frame $\left(\hat{\mathbf{t}}_{i j}, \hat{\mathbf{n}}_{i j}\right)$, as follows (Figure A1c):

$$
\mathbf{U}_{i}=\mathbf{V}_{i}+\mathbf{w}_{i} \times \mathbf{r}_{i}=\mathbf{V}_{i} \cdot \hat{\mathbf{n}}_{i j} \hat{\mathbf{n}}_{i j}+\left(\mathbf{V}_{i} \cdot \hat{\mathbf{t}}_{i j}+w_{i} r_{i}\right) \hat{\mathbf{t}}_{i j},
$$

where $\mathbf{O}_{i}$ and $\mathbf{V}_{i}$ are the position and velocity of the center of mass of disk $i, \mathbf{w}_{i}$ is its angular velocity, and $\mathbf{r}_{i}$ is the position vector of point $P_{i}$ relative to the center of mass $\mathbf{O}_{i}$ $\left(\left\|\mathbf{r}_{i}\right\|=r_{i}\right.$, where $r_{i}$ is the radius of disk $\left.i\right)$. The scalar $w_{i}$ gives the magnitude and direction of the angular velocity of disk $i$ (positive counterclockwise).

[91] A symmetric equation is written for the velocity $\mathbf{U}_{j}$ of the material point $P_{j}$ located in the perimeter of disk $j$, expressed in the local reference frame $\left(\hat{\mathbf{t}}_{j i}, \hat{\mathbf{n}}_{j i}\right)$ :

$$
\mathbf{U}_{j}=\mathbf{V}_{j}+\mathbf{w}_{j} \times \mathbf{r}_{j}=\mathbf{V}_{j} \cdot \hat{\mathbf{n}}_{j i} \hat{\mathbf{n}}_{j i}+\left(\mathbf{V}_{j} \cdot \hat{\mathbf{t}}_{j i}+w_{j} r_{j}\right) \hat{\mathbf{t}}_{j i} .
$$

[92] Consider a collision between disks $i$ and $j$. Then, we can calculate the change in velocity for each particle following the collision. Velocity changes for each disk are also expressed in the local reference frames linked to the contact point:

$$
\begin{aligned}
\Delta \mathbf{U}_{i}= & \mathbf{U}_{i}^{+}-\mathbf{U}_{i}^{-}=\left(\mathbf{V}_{i}^{+}-\mathbf{V}_{i}^{-}\right) \cdot \hat{\mathbf{n}}_{i j} \hat{\mathbf{n}}_{i j} \\
& +\left[\left(\mathbf{V}_{i}^{+}-\mathbf{V}_{i}^{-}\right) \cdot \hat{\mathbf{t}}_{i j}+\left(w_{i}^{+}-w_{i}^{-}\right) r_{i}\right] \hat{\mathbf{t}}_{i j} \\
\Delta \mathbf{U}_{j}= & \mathbf{U}_{j}^{+}-\mathbf{U}_{j}^{-}=\left(\mathbf{V}_{j}^{+}-\mathbf{V}_{j}^{-}\right) \cdot \hat{\mathbf{n}}_{j i} \hat{\mathbf{n}}_{j i} \\
& +\left[\left(\mathbf{V}_{j}^{+}-\mathbf{V}_{j}^{-}\right) \cdot \hat{\mathbf{t}}_{j i}+\left(w_{j}^{+}-w_{j}^{-}\right) r_{j}\right] \hat{\mathbf{t}}_{j i},
\end{aligned}
$$




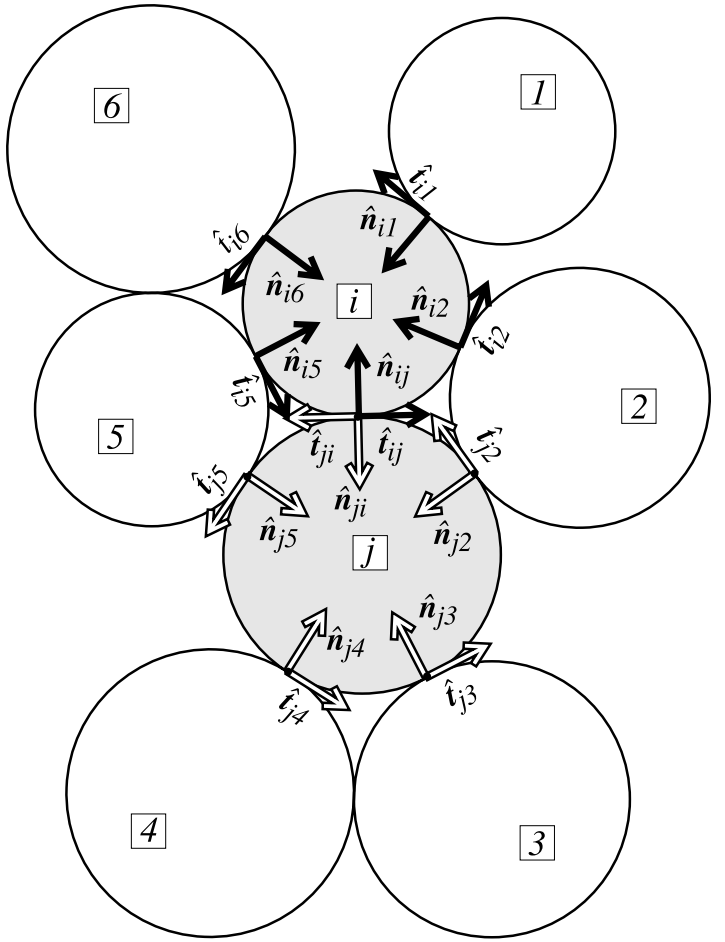

Figure A2. Particles susceptible to form a contact with disks $i$ and $j$, with their respective local reference frames. The sets of particles exerting contact forces on disks $i$ and $j$ are $k=\{1,2, j, 5,6\}$ and $l=\{2,3,4,5, i\}$, respectively (see text for explanation).

where the superscript minus and plus indicate velocities immediately before and after the collision, respectively.

[93] From these equations we calculate the variation of relative velocity $\Delta \mathbf{U}_{i j}$ between material points $P_{i}$ and $P_{j}$ resulting from the collision between the two disks:

$$
\begin{aligned}
\Delta \mathbf{U}_{i j} & =\Delta \mathbf{U}_{i j}^{+}-\Delta \mathbf{U}_{i j}^{-}=\left(\mathbf{U}_{i}^{+}-\mathbf{U}_{j}^{+}\right)-\left(\mathbf{U}_{i}^{-}-\mathbf{U}_{j}^{-}\right) \\
& =\left(\mathbf{U}_{i}^{+}-\mathbf{U}_{i}^{-}\right)-\left(\mathbf{U}_{j}^{+}-\mathbf{U}_{j}^{-}\right)=\Delta \mathbf{U}_{i}-\Delta \mathbf{U}_{j} .
\end{aligned}
$$

We calculate the components of $\Delta \mathbf{U}_{i j}$ in the local reference frame $\left(\hat{\mathbf{t}}_{i j}, \hat{\mathbf{n}}_{i j}\right)$ :

$$
\begin{gathered}
\Delta \mathbf{U}_{i j}=\Delta U_{i j}^{n} \hat{\mathbf{n}}_{i j}+\Delta U_{i j}^{t} \hat{\mathbf{t}}_{i j}, \\
\Delta U_{i j}^{n}=\left(\Delta \mathbf{V}_{i}-\Delta \mathbf{V}_{j}\right) \cdot \hat{\mathbf{n}}_{i j}, \\
\Delta U_{i j}^{t}=\left(\Delta \mathbf{V}_{i}-\Delta \mathbf{V}_{j}\right) \cdot \hat{\mathbf{t}}_{i j}+\left(\Delta w_{i} r_{i}+\Delta w_{j} r_{j}\right),
\end{gathered}
$$

where we have $\Delta \mathbf{V}_{i}=\mathbf{V}_{i}^{+}-\mathbf{V}_{i}^{-}, \Delta \mathbf{V}_{j}=\mathbf{V}_{j}^{+}-\mathbf{V}_{j}^{-}, \Delta w_{i}=w_{i}^{+}-$ $w_{i}^{-}$, and $\Delta w_{j}=w_{j}^{+}-w_{j}^{-}$.

\section{A2. Balance Equations}

[94] In this section, we shall use the balance of momenta to calculate the equations relating the contact forces and the relative velocities between particles at contact points. Consider an assembly of particles, which are moving as a result of large-scale deformation of the granular medium. Let $i$ and $j$ be two contacting disks at time $t$. We may calculate the variation of velocity of the particles as a function of the impulsions exerted on each particle. The total force acting on particle $i$ is given by (Figure A2)

$$
\mathbf{F}_{i}=\sum_{k}\left[N_{i k} \hat{\mathbf{n}}_{i k}+T_{i k} \hat{\mathbf{t}}_{i k}\right]+\mathbf{f}_{i}^{v},
$$

where $k$ represents the index of particles susceptible to be in contact with disk $i$. These neighboring particles may exert contact forces, which are decomposed according to their respective local reference frames: $N_{i k} \hat{\mathbf{n}}_{i k}$ and $T_{i k} \hat{\mathbf{t}}_{i k}$ are the normal and shear components of the contact force exerted by particle $k$ on particle $i$. $\mathbf{f}_{i}^{v}$ is the total bulk force acting on particle $i$ (e.g., the gravity force). In this representation, we assume that the internal stresses between different parts of the system are given by contact forces, and the external stresses exerted on the system are given by bulk or boundary forces.

[95] A similar expression holds for the force resultant exerted on particle $j$ :

$$
\mathbf{F}_{j}=\sum_{l}\left[N_{j l} \hat{\mathbf{n}}_{j l}+T_{j l} \hat{\mathbf{t}}_{j l}\right]+\mathbf{f}_{j}^{v}
$$

where $l$ represents the index of particles susceptible to be in contact with disk $j$. Note that disk $j$ is one of the close neighbors of disk $i$ and vice versa.

[96] Consider a small time interval $\Delta \tau$ over which the particles in the system may interact and collide. Integrating Newton's second law for particle $i$ during time interval $\Delta \tau$ yields

$$
\int_{\Delta \tau} \mathbf{F}_{i} d t=m_{i}\left[\mathbf{V}_{i}^{+}-\mathbf{V}_{i}^{-}\right]=m_{i} \Delta \mathbf{V}_{i}=\sum_{k}\left[R_{i k} \hat{\mathbf{n}}_{i k}+S_{i k} \hat{\mathbf{t}}_{i k}\right]+\mathbf{P}_{i}^{v},
$$

where

$$
\begin{aligned}
R_{i k} \hat{\mathbf{n}}_{i k} & =\int_{\Delta \tau} N_{i k} \hat{\mathbf{n}}_{i k} d t, \\
S_{i k} \hat{\mathbf{t}}_{i k} & =\int_{\Delta \tau} T_{i k} \hat{\mathbf{t}}_{i k} d t, \\
\mathbf{P}_{i}^{v} & =\int_{\Delta \tau} \mathbf{f}_{i}^{v} d t .
\end{aligned}
$$

Here, $R_{i k} \hat{\mathbf{n}}_{i k}, S_{i k} \hat{\mathbf{t}}_{i k}$ and $\mathbf{P}_{i}^{v}$ represent contact impulsions at contacts $(i k)$ and the bulk force impulsion acting on particle $i$, respectively. The variables $\mathbf{V}_{i}^{-}$and $\mathbf{V}_{i}^{+}$correspond to the instantaneous velocities of particle $i$ at the beginning and at the end of time interval $\Delta \tau . m_{i}$ is the mass of particle $i$. This equation states that the change of momentum is equal to the time integral of the contact and the bulk forces acting on the particle.

[97] A similar equation holds for particle $j$ :

$$
\begin{aligned}
\int_{\Delta \tau} \mathbf{F}_{j} d t & =m_{j}\left[\mathbf{V}_{j}^{+}-\mathbf{V}_{j}^{-}\right]=m_{j} \Delta \mathbf{V}_{j} \\
& =\sum_{l}\left[R_{j l} \hat{\mathbf{n}}_{j l}+S_{j l} \hat{\mathbf{t}}_{j l}\right]+\mathbf{P}_{j}^{v}
\end{aligned}
$$

[98] The variation of the angular velocity of disk $i$ is calculated by relating angular momentum and torque. Con- 
sidering an inertial coordinate system whose origin is located at the center of mass $\mathbf{O}_{i}$ of disk $i$, we have

$$
\boldsymbol{\Gamma}_{i}=\sum_{k} \mathbf{r}_{i k} \times T_{i k} \hat{\mathbf{t}}_{i k}
$$

where $\boldsymbol{\Gamma}_{i}$ is the torque exerted on particle $i$, and $\mathbf{r}_{i k}$ is the position vector of the contact point relative to $\mathbf{O}_{i}\left(\left\|\mathbf{r}_{i k}\right\|=r_{i}\right)$. Note that only shear forces apply torque about the center of mass, since they are perpendicular to the position vector $\mathbf{r}_{i k}$. The bulk force $\mathbf{f}_{i}^{v}$ does not exert any torque since it acts at the center of mass $\mathbf{O}_{i}$. The torque may be linked to the variation of angular velocity in $2 \mathrm{D}$ by means of the following expression

$$
\begin{aligned}
\int_{\Delta \tau} \Gamma_{i} d t & =I_{i}\left[\mathbf{w}_{i}^{+}-\mathbf{w}_{i}^{-}\right]=I_{i} \Delta \mathbf{w}_{i} \\
& \Rightarrow I_{i} \Delta w_{i}=r_{i} \sum_{k} S_{i k},
\end{aligned}
$$

where $I_{i}$ is the moment of inertia of disk $i$ about an axis passing by the center of mass, and $I_{i} \Delta w_{i}$ is the variation of the spin angular momentum of disk $i$.

[99] A similar equation holds for disk $j$ :

$$
\begin{aligned}
\int_{\Delta \tau} \Gamma_{j} d t & =I_{j}\left[\mathbf{w}_{j}^{+}-\mathbf{w}_{j}^{-}\right]=I_{j} \Delta \mathbf{w}_{j} \\
& \Rightarrow I_{j} \Delta w_{j}=r_{j} \sum_{l} S_{j l} .
\end{aligned}
$$

We can now calculate the equations relating the variation of relative velocity $\Delta \mathbf{U}_{i j}$ between material points $P_{i}$ and $P_{j}$, and the impulsions acting on disks $i$ and $j$ in time interval $\Delta \tau$. Replacing equations (A11) and (A13) in equation (A7), we obtain:

$$
\begin{aligned}
\Delta U_{i j}^{n}= & \frac{R_{i j}}{m_{i j}^{n}}+\frac{1}{m_{i}}\left[\sum_{k(\neq j)}\left[R_{i k} \hat{\mathbf{n}}_{i k}+S_{i k} \hat{\mathbf{t}}_{i k}\right]+\mathbf{P}_{i}^{v}\right] \cdot \hat{\mathbf{n}}_{i j} \\
& -\frac{1}{m_{j}}\left[\sum_{l(\neq i)}\left[R_{j l} \hat{\mathbf{n}}_{j l}+S_{j l} \hat{\mathbf{t}}_{j l}\right]+\mathbf{P}_{j}^{v}\right] \cdot \hat{\mathbf{n}}_{i j},
\end{aligned}
$$

where

$$
\frac{1}{m_{i j}^{n}}=\frac{1}{m_{i}}+\frac{1}{m_{j}}
$$

Note that the sums over indices $k$ and $l$ do not include the contact between disks $i$ and $j$. This expression yields the impulsion $R_{i j}$ normal to the contact:

$$
R_{i j}=m_{i j}^{n} \Delta U_{i j}^{n}+K_{i j}^{n}
$$

with

$$
\begin{aligned}
\frac{K_{i j}^{n}}{m_{i j}^{n}}= & -\frac{1}{m_{i}}\left[\sum_{k(\neq j)}\left[R_{i k} \hat{\mathbf{n}}_{i k}+S_{i k} \hat{\mathbf{t}}_{i k}\right]+\mathbf{P}_{i}^{v}\right] \cdot \hat{\mathbf{n}}_{i j} \\
& +\frac{1}{m_{j}}\left[\sum_{l(\neq i)}\left[R_{j l} \hat{\mathbf{n}}_{j l}+S_{j l} \hat{\mathbf{t}}_{j l}\right]+\mathbf{P}_{j}^{v}\right] \cdot \hat{\mathbf{n}}_{i j} .
\end{aligned}
$$

The same procedure can be used to express the tangential component of the relative velocity as a function of the impulsion, by using equations (A11), (A13), (A15) and (A16) in equation (A8):

$$
\begin{aligned}
\Delta U_{i j}^{t}= & \frac{S_{i j}}{m_{i j}^{t}}+\frac{1}{m_{i}}\left[\sum_{k(\neq j)}\left[R_{i k} \hat{\mathbf{n}}_{i k}+S_{i k} \hat{\mathbf{t}}_{i k}\right]+\mathbf{P}_{i}^{v}\right] \cdot \hat{\mathbf{t}}_{i j} \\
& -\frac{1}{m_{j}}\left[\sum_{l(\neq i)}\left[R_{j l} \hat{\mathbf{n}}_{j l}+S_{j l} \hat{\mathbf{t}}_{j l}\right]+\mathbf{P}_{j}^{v}\right] \cdot \hat{\mathbf{t}}_{i j} \\
& +\frac{r_{i}^{2}}{I_{i}} \sum_{k(\neq j)} S_{i k}+\frac{r_{j}^{2}}{I_{j}} \sum_{l(\neq i)} S_{j l},
\end{aligned}
$$

where

$$
\frac{1}{m_{i j}^{t}}=\frac{1}{m_{i}}+\frac{1}{m_{j}}+\frac{r_{i}^{2}}{I_{i}}+\frac{r_{j}^{2}}{I_{j}} .
$$

Equation (A22) allows us to calculate the impulsion $S_{i j}$ in $\hat{\mathbf{t}}_{i j}$ direction:

$$
S_{i j}=m_{i j}^{t} \Delta U_{i j}^{t}+K_{i j}^{t}
$$

with

$$
\begin{aligned}
\frac{K_{i j}^{t}}{m_{i j}^{t}}= & -\frac{1}{m_{i}}\left[\sum_{k(\neq j)}\left[R_{i k} \hat{\mathbf{n}}_{i k}+S_{i k} \hat{\mathbf{t}}_{i k}\right]+\mathbf{P}_{i}^{v}\right] \cdot \hat{\mathbf{t}}_{i j} \\
& +\frac{1}{m_{j}}\left[\sum_{l(\neq i)}\left[R_{j l} \hat{\mathbf{n}}_{j l}+S_{j l} \hat{\mathbf{t}}_{j l}\right]+\mathbf{P}_{j}^{v}\right] \cdot \hat{\mathbf{t}}_{i j} \\
& -\frac{r_{i}^{2}}{I_{i}} \sum_{k(\neq j)} S_{i k}-\frac{r_{j}^{2}}{I_{j}} \sum_{l(\neq i)} S_{j l} .
\end{aligned}
$$

\section{A3. Energy Dissipation}

[100] The conservation of momentum does not provide by itself a complete description of the equations of motion of the particle set. Indeed, the interactions between particles may lead to a loss of mechanical energy as a result of work done by nonconservative forces such as friction. Energy dissipation during a collision event will induce a reduction of the kinetic energy of the colliding particles. In view of the fact that the principal unknown variables are velocities, it is simpler to consider an additional constraint relating velocities before and after collisions. On phenomenological grounds, a very simple approach used in the framework of the CD method is to introduce restitution coefficients defined by [Moreau, 1993]:

$$
\begin{aligned}
U_{i}^{n+}-U_{j}^{n+} & =-\rho_{n}\left(U_{i}^{n-}-U_{j}^{n-}\right) \\
& \Rightarrow U_{i j}^{n+}=-\rho_{n} U_{i j}^{n-},
\end{aligned}
$$

where $\rho_{n}$ is the Newton coefficient of normal restitution. The variables $U_{i}^{n-}$ and $U_{i}^{n+}$ indicate the normal component of velocity of particle $i$ at the contact point, immediately before and after a collision, respectively. This equation states that the relative normal velocities before and after a collision between two disks are proportional and have 


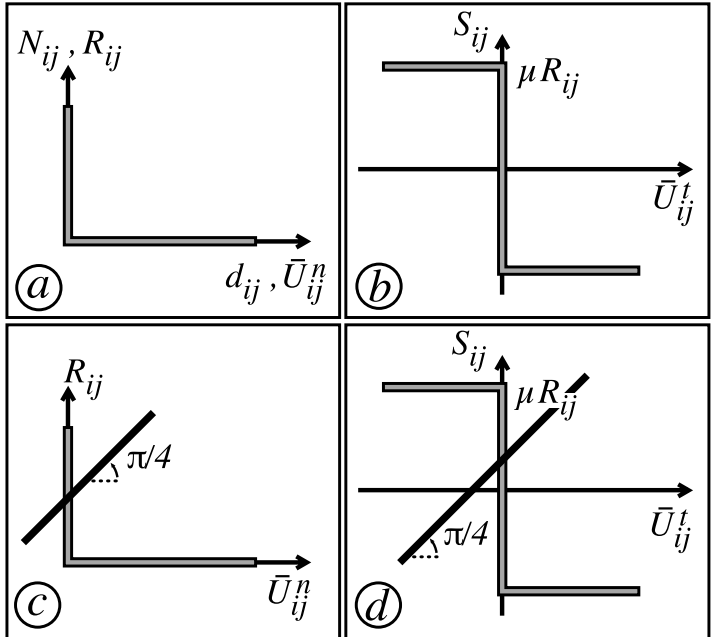

Figure A3. (a) Graph illustrating the Signorini condition in the direction normal to the contact between disks $i$ and $j$. (b) Graph illustrating the Coulomb friction law at the contact $i j$, relating the formal relative tangential velocity $\bar{U}_{i j}^{t}$ and the impulsion $S_{i j}$ in the tangential direction. (c) Solution of the equations of motion in the normal direction, obtained by the intersection between the Signorini graph in velocity and the line relating normal impulsion and the formal relative normal velocity. (d) Solution of the equations of motion in the tangential direction, obtained by the intersection between the Coulomb law and the line relating tangential impulsion and normal relative tangential velocity.

opposite directions. We have $\rho_{n} \in[0,1]$, where $\rho_{n}=0$ corresponds to a perfectly inelastic collision (particles stick together after the collision), and $\rho_{n}=1$ corresponds to a perfectly elastic collision involving no energy dissipation.

[101] A similar equation can be written for the tangential component of velocity along a contact:

$$
\begin{aligned}
U_{i}^{t+}-U_{j}^{t+} & =-\rho_{t}\left(U_{i}^{t-}-U_{j}^{t-}\right) \\
& \Rightarrow U_{i j}^{t+}=-\rho_{t} U_{i j}^{t-},
\end{aligned}
$$

where $\rho_{t}$ is the coefficient of tangential restitution. The variables $U_{i}^{t-}$ and $U_{i}^{t+}$ indicate the tangential component of velocity before and after a collision, respectively. This equation states that the relative tangential velocities between the contact points $P_{i}$ and $P_{j}$, are proportional and have opposite directions before and after a collision. Again, we have $\rho_{t} \in[0,1]$, where $\rho_{t}=0$ corresponds to an inelastic collision (in the tangential direction). The limit $\rho_{t}=1$ implies that the tangential relative velocity has the same magnitude and an opposite direction after the collision.

[102] A major issue is the detection of the collisions between particles during the evolution of the particle set. For the sake of clarity we analyze separately the mechanical interactions between the colliding particles in the normal and tangential directions $\left(\hat{\mathbf{n}}_{i j}, \hat{\mathbf{t}}_{i j}\right)$. Thus we will distinguish the normal collisions from the tangential ones, each of which will have a different physical interpretation.

\section{A3.1. Collision in the "Normal" Direction $\hat{\mathbf{n}}_{i j}$}

[103] The concept of collision in the normal direction $(\mathrm{CN})$ refers to any two particles that are exerting contact normal forces on each other, whether or not they are truly colliding dynamically. Thus different kinds of $\mathrm{CN}$ should be distinguished:

[104] 1. Dynamic collisions in the normal direction (DCN) occur at very short timescales, much smaller than the characteristic evolution time of the system. In this situation, the normal relative velocity before the collision is negative: $U_{i j}^{n-}<0$, which according to our sign conventions means that the particles are getting closer to each other. The normal relative velocity after a DCN event must satisfy the condition $U_{i j}^{n+} \geq 0$. This condition is necessary to prevent interpenetration between particles. To simplify the analysis we will consider that dynamic collisions are instantaneous and that they may generate discontinuities in the velocity field and an instantaneous propagation of impulses within the particle set. This last assertion is valid both in the normal and tangential directions.

[105] 2. Particles that are in contact persistently over a finite time interval $\tau$ may also be colliding in the normal direction, since they may exert normal impulse on each other. A persistent contact implies that the instantaneous relative velocity $U_{i j}^{n}=0$ during time interval $\tau$. Persistent contacts may open during multiple collision events. This situation may be expressed in terms of normal relative velocities at the beginning and at the end of a small time interval $\Delta \tau: U_{i j}^{n-}=0$ and $U_{i j}^{n+}>0$ (note that the normal velocity field is discontinuous over time interval $\Delta \tau$ ).

[106] A collision between two disks $i$ and $j$ in time interval $\Delta \tau$ requires that particles should be in contact at the beginning of the time interval, otherwise contact forces are nonexistent. This condition may be expressed as a relation between the distance $d_{i j}$ and the normal force $N_{i j}$ known as the Signorini condition. This condition can be formulated by means of the following inequalities:

$$
\begin{gathered}
d_{i j} \geq 0, \\
N_{i j} \geq 0, \\
d_{i j} N_{i j}=0 .
\end{gathered}
$$

Figure A3a illustrates the graph of the displacement Signorini condition. These relations state that the distance between particles is positive or zero; if the distance is positive then the contact normal force is nonexistent, and if the distance is zero then particles are in contact and the normal force can take any nonnegative value (with the convention that positive normal forces are repulsive). This condition imposes that particles do not interpenetrate as a consequence of repulsive normal forces.

[107] The contact between the particles is not a sufficient condition for a $\mathrm{CN}$ to take place (remember that a $\mathrm{CN}$ event implies contact normal force interactions between particles). Particles in contact may collide or not in the normal direction, depending on their relative velocities at the contact point. From the definition of the coefficient of normal restitution in equation (A25), we can state that a collision in the normal direction occurs if and only if the following condition is verified:

$$
U_{i j}^{n+}+\rho_{n} U_{i j}^{n-}=0 .
$$


During a DCN the relative normal velocity has opposite directions before and after the collision event (with $\rho_{n}>0$ ).

[108] Hence let us define a new variable to which we will refer as the formal relative normal velocity (FRNV), such that

$$
\bar{U}_{i j}^{n}=m_{i j}^{n}\left(U_{i j}^{n+}+\rho_{n} U_{i j}^{n-}\right) .
$$

We have $\bar{U}_{i j}^{n}=0$ if and only if there is a collision in the normal direction between particles. The expression of $\bar{U}_{i j}^{n}$ given in equation (A29) differs from that given by Moreau [1993] by the positive factor $m_{i j}^{n}$ (see equation (A18)), which will allow to simplify the solution of the equations in section A4. The $\mathrm{CN}$ event may be dynamic or it may correspond to a persistent contact during time interval $\Delta \tau$ (in both settings $\bar{U}_{i j}^{n}=0$ ). The situation in which a persistent contact opens as a consequence of a multiple collision is such that $\bar{U}_{i j}^{n}>0$. This inequality must be satisfied to prevent interpenetration. Moreover, repulsive normal forces exerted along the contacts induce positive normal velocities between particles. Note that the opening of a persistent contact is not considered as a $\mathrm{CN}$ event and contact forces are zero.

[109] The previous relations may be expressed by the following inequalities, which relate the FRNV and the normal impulsion (once particles are in contact):

$$
\begin{gathered}
\bar{U}_{i j}^{n} \geq 0, \\
R_{i j} \geq 0, \\
\bar{U}_{i j}^{n} R_{i j}=0 .
\end{gathered}
$$

These inequalities are known as the Signorini condition in velocity, and they define the possible values for variables $\bar{U}_{i j}^{n}$ and $R_{i j}$, as illustrated in the graph in Figure A3a: The FRNV between particles is positive or zero; if the FRNV is positive then the normal impulsion is nonexistent, and if the FRNV is zero then the particles collide in the normal direction and the normal impulsion can take any nonnegative value.

\section{A3.2. Collision in the "Tangential" Direction $\hat{\mathbf{t}}_{\boldsymbol{i} j}$}

[110] A similar analysis may be carried out for tangential collisions. The tangential relative velocity at the contact point determines the magnitude and the direction of the frictional forces and impulsions acting in the contact plane. In general, we may assume that the friction force $T_{i j}$ is directed opposite to the instantaneous relative velocity $U_{i j}^{t}$. The dependence between the magnitudes of $T_{i j}$ and $U_{i j}^{t}$ may be specified by simple relations: If $U_{i j}^{t} \neq 0$ then the particles slip along the contact and we have $\left\|T_{i j}\right\|=\mu N_{i j}$, where $\mu$ is the coefficient of friction; if $U_{i j}^{t}=0$ then the contact is nonslipping and $\left\|T_{i j}\right\| \leq \mu N_{i j}$.

[111] From the definition of the coefficient of tangential restitution we may establish the relationship between shear forces and relative velocities during a collision. We first discuss the physical interpretation of a collision in the tangential direction, which should satisfy the following equation:

$$
U_{i j}^{t+}+\rho_{t} U_{i j}^{t-}=0
$$

Equation (A31) results from the phenomenological relation given in equation (A26).

[112] Consider a collision event in which particles are rotating with respect to each other immediately before the collision (e.g., $U_{i j}^{t-} \neq 0$ ). The collision in the tangential direction (CT) may be dynamic or it may correspond to a persistent contact during time interval $\Delta \tau$. Equation (A31) implies that the relative tangential velocity has opposite direction before and after the collision (if $\rho_{t}>0$ and $U_{i j}^{t-} \neq$ $0)$. The precise interaction between particles during a CT event may be complex. Nevertheless, we can state that the instantaneous relative velocity $U_{i j}^{t}=0$ at a given moment during the collision process. This key observation suggests that the "mean" tangential relative velocity during the collision event is close to zero. We may then assert that the mean status of the contact between the two particles is close to a nonslipping condition. Thus the concept of collision in the tangential direction as formulated by means of the restitution coefficient may be related to a nonslipping shear interaction along the contact (equations (A26) and (A31)).

[113] To simplify the analysis, let us define a new variable to which we will refer as the formal relative tangential velocity (FRTV), such that

$$
\bar{U}_{i j}^{t}=m_{i j}^{t}\left(U_{i j}^{t+}+\rho_{t} U_{i j}^{t-}\right) .
$$

We will assume that $\bar{U}_{i j}^{t}=0$ if and only if the mean status of the contact is nonslipping $\left(m_{i j}^{t}\right.$ is a positive constant defined in equation (A22)).

[114] Consider a collision event in which particles form a nonslipping persistent contact $\left(U_{i j}^{t}=0\right.$ and $\left.\bar{U}_{i j}^{t}=0\right)$. In this situation, the particles roll without slipping and shear forces are below the maximum frictional resistance. This contact behavior is consistent with the definition of collision in the tangential direction, satisfying equation (A32). The nonslipping condition along persistent contacts may be lost during multiple collision events. This situation may be expressed in terms of tangential relative velocities at the beginning and at the end of a small time interval $\Delta \tau: U_{i j}^{t-}=$ $0, U_{i j}^{t+} \neq 0$, and $\bar{U}_{i j}^{t}=m_{i j}^{t} U_{i j}^{t+}$ (note that the tangential velocity field is discontinuous over the time interval $\Delta \tau$ ). Particles will then slip along the contact, and the friction force will reach its maximum value. In this situation, the FRTV will be oriented in the same direction as $U_{i j}^{t+}$.

[115] The relationship between the FRTV and the impulsion associated with shear (frictional) forces may be summarized by the following inequalities (Figure A3b):

$$
\begin{gathered}
\bar{U}_{i j}^{t}=0 \Rightarrow S_{i j} \in\left[-\mu R_{i j}, \mu R_{i j}\right], \\
\bar{U}_{i j}^{t}>0 \Rightarrow S_{i j}=-\mu R_{i j}, \\
\bar{U}_{i j}^{t}<0 \Rightarrow S_{i j}=\mu R_{i j} .
\end{gathered}
$$

If $\bar{U}_{i j}^{t}=0$, we assume that there is no slip between the particles at the contact point and the tangential impulsion can take any value in between a negative and a positive threshold. The particles may roll without slipping and without dissipating energy by friction.

[116] If $\bar{U}_{i j}^{t} \neq 0$, then the particles slip along the contact, and the friction force attains its threshold value. Note that 
this physical situation is not rigorously considered as a CT event, even though frictional interactions are present. This simple law is equivalent to a Coulomb friction law in which the shear force $T_{i j} \in\left[-\mu N_{i j}, \mu N_{i j}\right]$.

\section{A4. Solution of the Equations of Motion}

[117] To solve the equations of motion for the set of particles, we express the relative velocities in terms of formal relative velocities and restitution coefficients in the equations of dynamics. The relative normal velocity is given by

$$
\Delta U_{i j}^{n}=U_{i j}^{n+}-U_{i j}^{n-}=\frac{\bar{U}_{i j}^{n}}{m_{i j}^{n}}-\left(1+\rho_{n}\right) U_{i j}^{n-} .
$$

Inserting (A34) in equation (A19) yields

$$
R_{i j}=\bar{U}_{i j}^{n}+\left[K_{i j}^{n}-m_{i j}^{n}\left(1+\rho_{n}\right) U_{i j}^{n-}\right] .
$$

This is a linear equation between the variables $\bar{U}_{i j}^{n}$ and $R_{i j}$, with unit (positive) slope. The offset term depends on the mass of particles $i$ and $j$, the coefficient of normal restitution, the relative velocity before the collision and the forces acting on the contacts other than $i j$.

[118] The intersection between this line and the graph relating normal impulsion and FRNV is unique and defines the solution for the impulsion and velocity in the normal direction for particles that are in contact at the beginning of time interval $\Delta \tau$ (Figure A3c). In a similar way, the relative tangential velocity is given by

$$
\Delta U_{i j}^{t}=U_{i j}^{t+}-U_{i j}^{t-}=\frac{\bar{U}_{i j}^{t}}{m_{i j}^{t}}-\left(1+\rho_{t}\right) U_{i j}^{t-} .
$$

Inserting equation (A36) into equation (A23) yields

$$
S_{i j}=\bar{U}_{i j}^{t}+\left[K_{i j}^{t}-m_{i j}^{t}\left(1+\rho_{t}\right) U_{i j}^{t-}\right] .
$$

This is also a linear equation with unit (positive) slope, between variables $\bar{U}_{i j}^{t}$ and $S_{i j}$. The offset term depends on the mass of particles $i$ and $j$, the coefficient of tangential restitution, the relative velocity before the collision and the forces acting on the contacts other than $i j$. The intersection between this line and the graph relating tangential impulsion and FRTV is unique, and corresponds to the solution for the impulsion and velocity in the tangential direction for particles that are in contact at the beginning of time interval $\Delta \tau$ (Figure A3d).

[119] Let $N_{p}$ be the number of particles in the system and $N_{c}$ be the total number of contacts. The solution of the set of equations in $2 \mathrm{D}$ at any time $\tau$ consists in calculating the impulsions acting on the particles during a small time interval $\Delta \tau$, and the velocities at time $\tau+\Delta \tau$. These unknown variables are calculated from the knowledge of the initial positions and velocities of particles at time $\tau$. The number of unknown variables is $3 N_{p}+2 N_{c}$, since each particle involves two translational velocities and an angular velocity, and each contact has two unknown force components (shear and normal forces). The number of available equations is also $3 N_{p}+2 N_{c}$ : To each contact correspond two Signorini graphs and each particle satisfies three dynamic equations (in integral form). Thus in principle the system of equations may be solved.

[120] From a practical point of view, the calculation of the unknown variables is done following several steps. From the Signorini graphs and the linear equations relating impulses and formal velocities, we calculate the contact forces. The algorithm uses an implicit-type time stepping in which we begin with an arbitrary distribution of impulsions $R_{i j}$ and $S_{i j}$, which may correspond to their values in the previous time step. For each contact $i j$ we may calculate the linear equation relating impulsion and relative formal velocity. The intersection between the linear equation and the Signorini graph (Figure A3), gives a set of new values of the impulsions $R_{i j}^{\prime}$ and $S_{i j}^{\prime}$, which may be compared with the previous ones. This process is iterated until a convergence criterion is satisfied (e.g., $\left|R_{i j}^{\prime}-R_{i j}<\epsilon\right|$, where $\epsilon \ll 1$ ).

[121] Once the impulsions and formal velocities are known, we can calculate the contact forces acting on each particle during time interval $\Delta \tau$ :

$$
\begin{aligned}
& N_{i j} \cong R_{i j} / \Delta \tau \\
& T_{i j} \cong S_{i j} / \Delta \tau .
\end{aligned}
$$

The positions and velocities at the end of time interval $\Delta \tau$ may be calculated from the definition of relative formal velocities or from the total impulse acting on each particle.

\section{Notation}

$i, j$ indices indicating two arbitrary disks in the particle assembly.

$P_{i}, P_{j} \quad$ material points located on the perimeter of the disks and coinciding with the contact point.

$\hat{\mathbf{t}}_{i j}, \hat{\mathbf{n}}_{i j}$ unitary vectors defining the local reference frame at the contact between disks $i$ and $j$.

$T, N$ shear and normal contact forces.

$T_{i j}, N_{i j}$ magnitudes of the shear and normal forces applied at the contact $i j$.

$U^{n}, U^{t}$ relative normal and tangential velocities between two particles.

$\mathbf{U}_{i}, \mathbf{U}_{j} \quad$ velocities of the material points $P_{i}$ and $P_{j}$.

$\mathbf{O}_{i}, \mathbf{O}_{j}$ positions of the center of mass of disks $i$ and $j$.

$\mathbf{V}_{i}, \mathbf{V}_{j} \quad$ velocities of the center of mass of disks $i$ and $j$.

$\mathbf{w}_{i}, \mathbf{w}_{j}$ vectors indicating angular velocities of disks $i$ and $j$.

$w_{i}, w_{j}$ magnitude of angular velocities of disks $i$ and $j$.

$\mathbf{r}_{i}$ position vector of point $P_{i}$ relative to the center of mass $\mathbf{O}_{i}$.

$\mathbf{r}_{j}$ position vector of point $P_{j}$ relative to the center of mass $\mathbf{O}_{j}$.

$r_{i}, r_{j}$ radius of disks $i$ and $j$.

$\Delta \mathbf{U}_{i}, \Delta \mathbf{U}_{j} \quad$ velocity changes at contact points $P_{i}$ and $P_{j}$ during a collision.

$\Delta \mathbf{U}_{i j}$ variation of relative velocity between material points $P_{i}$ and $P_{j}$ resulting from the collision between disks. 
$\Delta U_{i j}^{n}, \Delta U_{i j}^{t} \quad$ normal and tangential components of $\Delta \mathbf{U}_{i j}$ in the local reference frame.

$\mathbf{F}_{i}$ total force acting on particle $i$.

$\mathbf{f}_{i}^{v}$ total bulk force acting on particle $i$ (e.g., the gravity force).

$m_{i}, m_{j} \quad$ mass of particles $i$ and $j$.

$R_{i k}, S_{i k}$ contact impulsions exerted by particle $k$ on particle $i$ in the normal and tangential directions.

$\mathbf{P}_{i}^{v} \quad$ impulsion exerted by the bulk forces on particle $i$.

$\Gamma_{i}$ torque exerted on particle $i$.

$I_{i}$ moment of inertia of disk $i$ about an axis passing by the center of mass.

$K_{i j}^{n}, K_{i j}^{t} \quad$ constant coefficients in the linear expressions defining the local impulsions at contact $i j$.

$\rho_{n}, \rho_{t}$ coefficients of normal and tangential restitution.

$\mathrm{CN}$ collision in the normal direction.

DCN dynamic collision in the normal direction.

FRNV formal relative normal velocity.

CT collision in the tangential direction.

FRTV formal relative tangential velocity.

$\tau, \Delta \tau$ finite and differential time interval.

$d_{i j} \quad$ distance between particles $i$ and $j$.

$\bar{U}_{i j}^{n}, \bar{U}_{i j}^{t} \quad$ formal relative normal and tangential velocities at contact $i j$.

$\epsilon$ small positive value defining the convergence criterion for numerical solution.

$N_{p}, N_{c}$ number of particles and contacts in the system.

$\left(R_{\mathrm{Min}}, R_{\mathrm{Max}}\right)$ minimum and maximum particle radii in the grain size distribution.

$C_{u}$ uniformity coefficient of the grain size distribution.

$D_{10}, D_{60} \quad$ grain size diameter corresponding to $10 \%$ finer and $60 \%$ finer in the grain size distribution.

$U$ potential energy function for the construction of the numerical sample.

$g$ acceleration of gravity.

$\hat{z} \quad$ unit vector pointing upward.

$\left[P_{1}, \ldots, P_{n}\right]$ chain of particles located on the top of the particle set.

$\nu$ packing fraction of the sample.

$\bar{N}$ average coordination number of the sample.

$\rho$ density of the particles.

$h, l$ height and the width of the sample.

$\mu_{d d}$ friction coefficient between the disks.

$\mu_{d l}$ friction coefficient between the disks and the walls.

$\sigma_{c}$ horizontal stress applied along the right vertical wall during compaction of the sample.

$\sigma_{3}$ confining pressure applied on the sample boundaries.

$\epsilon_{z}, \Delta \epsilon_{z} \quad$ vertical strain of the sample and vertical strain interval.

$\Delta z \quad$ axial displacement of the top horizontal wall (positive in the direction of compression). $h_{0} \quad$ initial height of the sample.

$\sigma_{1}$ compressional axial stress applied on the sample.

DW biaxial test such that the confining pressure is applied through deformable walls.

$I$ inertia parameter.

$\dot{\gamma}$ shear rate.

$V_{s p}$ shortening displacement rate during the shear phase.

$w_{s b}$ mean width of the active shear bands.

DWL, DWH biaxial tests for low or high friction $\left(\mu_{d d}=\right.$ 0.3 and $\mu_{d d}=3$, respectively).

$\sigma_{1}^{\text {DWL }}$ compressional stress for low friction test.

$\bar{\sigma}_{\max }$ maximum value of the mean vertical stress, for a mobile interval $\Delta \epsilon_{z}=0.002$.

$\bar{\sigma}_{\text {res }}$ residual strength given by the mean vertical stress for $\epsilon_{z} \in[0.05,0.1]$.

$\bar{\sigma}_{\max }^{\mathrm{DWL}}, \bar{\sigma}_{\max }^{\mathrm{DWH}}$ maximum vertical stress for low and high friction.

$\bar{\sigma}_{\text {res }}^{\mathrm{DWL}}, \bar{\sigma}_{\text {res }}^{\mathrm{DWH}}$ residual strength for low and high friction.

$\Delta V_{s}^{\mathrm{DWL}}$ volumetric strain of the sample for low friction.

$\Delta V_{s}^{\mathrm{DWH}}$ volumetric strain for high friction.

$V$ volume of the sample.

$\mathrm{RCL}, \mathrm{RCH}$ number of rolling contacts for low and high friction.

SCL, SCH number of slipping contacts for low and high friction.

NCL, NCH number of contacts for low and high friction.

ZCL, ZCH number of contacts with zero force.

$\mu$ coefficient of friction between particles.

$\alpha_{i j}$ angle between the normal contact force and the mean direction of a force chain.

$\Delta x_{i j}$ distance between the centers of the disks, measured perpendicularly to the axis of the force chain.

$p$ mean stress $\left(\sigma_{1}+\sigma_{3}\right) / 2$.

$q$ deviatoric stress $\left(\sigma_{1}-\sigma_{3}\right) / 2$.

$\mu_{\text {macro, }} \mu_{\text {macro }}^{\text {res }}$ friction coefficients characterizing the macroscopic peak and residual strength.

$\phi$ internal friction angle.

[122] Acknowledgments. We would like to thank David Mainprice, James Hazzard, and an anonymous reviewer for helpful comments on the manuscript and Anne Delplanque for her technical help. This work was partially funded by ECOS-Nord/Colciencias project C00U01 FranceColombia 2002; CNRS program "Approche multiéchelles des propriétés macroscopiques des matériaux de structure", project 7, 2002; and INSU ACI PCN 2002

\section{References}

Abe, S., J. H. Dieterich, P. Mora, and D. Place (2002), Simulation of the influence of rate- and state-dependent friction on the macroscopic behavior of complex fault zones with the lattice solid model, Pure Appl. Geophys., 159, $1967-1983$.

Alshibli, K., and S. Sture (2000), Shear band formation in plane strain experiments of sand, J. Geotech. Geoenviron. Eng., 126, 495-503.

Bagnold, R. A. (1941), The physics of blown sand and desert dunes, Methuen and Co., 256 pp., CRC Press, Boca Raton, Fla.

Baotang, S., O. Stephansson, H. Einstein, and B. Ghahreman (1995), Coalescence of fractures under shear stresses in experiments, J. Geophys. Res., 100, 5975-5990.

Bardet, J. P. (1997), Experimental Soil Mechanics, 583 pp., Prentice-Hall, Upper Saddle River, N. J. 
Bardet, J. P., and J. Proubet (1992), Numerical simulations of shear bands in idealized granular materials, Solid State Phenomena, 23-24, 473482

Biegel, R. L., C. G. Sammis, and J. H. Dieterich (1989), The frictional properties of a simulated gouge having a fractal particle distribution, J. Struct. Geol., 11, 827-846.

Calvetti, F., G. Combe, and J. Lanier (1997), Experimental micromechanical analysis of a 2D granular material: Relation between structure evolution and loading path, Mech. Cohesive Frict. Mater., 2, $121-163$

Cambou, B. (1993), From global to local variables in granular materials, in Powders and Grains 93, edited by C. Thornton, pp. 73-86, A. A Balkema, Brookfield, Vt.

Chevoir, F., M. Prochnow, J. T. Jenkins, and P. Mills (2001), Dense granular flows down and inclined plane, in Powders and Grains 2001, edited by Y. Kishino, pp. 373-376, A. A. Balkema, Brookfield, $\mathrm{Vt}$.

Coduto, D. P. (1999), Geotechnical Engineering: Principles and Practices, 759 pp., Prentice-Hall, Upper Saddle River, N. J.

Cruden, D. M., and D. J. Varnes (1996), Landslide types and processes, in Landslides: Investigation and Mitigation, edited by A. K. Turner and R. L. Schuster, Spec. Rep. 247, pp. 36-75, Transp. Res. Board, Natl. Res. Counc., Washington, D. C.

Cundall, P. A., and O. D. L. Stack (1979), A discrete numerical model for granular assemblies, Geotechnique, 29(1), 47-65.

Cundall, P. A., A. Drescher, and O. D. L. Strack (1982), Numerical experiments on granular assemblies: Measurements and observations, in Proceedings of IUTAM Conference on Deformation and Failure of Granular Materials, edited by P. A. Vermeer and H. J. Luger, pp. 355-370, A. A. Balkema, Brookfield, Vt.

Dantu, P. (1957), Contribution à l'étude mécanique et géométrique des milieux pulvérulents, in Proceedings of the 4th International Conference on Soil Mechanics and Foundation Engineering, vol. 1, pp. 144-148, Butterworths Sci., London.

Daudon, D., J. Lanier, and M. Jean (1997), A micromechanical comparison between experimental results and numerical simulation of a biaxial test on 2D granular material, in Powders and Grains 1997, edited by R. P. Behringer and J. T. Jenkins, pp. 219-222, A. A. Balkema, Brookfield,

Desrues, J., R. Chambon, M. Mokni, and F. Mazerolle (1996), Void ratio evolution inside shear bands in triaxial sand specimens studied by computed tomography, Geotechniques, 46(3), 529-546.

Dieterich, J. H. (1979), Modeling of rock friction 1. Experimental results and constitutive equations, J. Geophys. Res., 84, 2161-2175.

Friedmann, S. J., G. Kwon, and W. Losert (2003), Granular memory and its effect on the triggering and distribution of rock avalanche events, J. Geophys. Res., 108(B8), 2380, doi:10.1029/2002JB002174

Frye, K. M., and C. Marone (2002), The effect of particle dimensionality on granular friction in laboratory shear zones, Geophys. Res. Lett., 29(19), 1916, doi:10.1029/2002GL015709.

Hazzard, J. F., R. P. Young, and S. C. Maxwell (2000), Micromechanical modeling of cracking and failure in brittle rocks, J. Geophys. Res., 105, $16,683-16,697$

Jaeger, H. M., S. R. Nagel, and R. P. Behringer (1996), Granular solids, liquids, and gases, Rev. Mod. Phys., 68(4), 1259-1273.

Jean, M. (1995), Frictional contact in collections of rigid or deformable bodies: Numerical simulation of geomaterials, in Mechanics of Geomaterial Interfaces, edited by A. P. S. Selvadurai and M. J. Boulon, pp. 453-486, Elsevier, New York.

Jean, M. (1999), The non smooth contact dynamics method, in Comput. Methods Appl. Mech. Eng., 177, 235-257.

Jean, M. (2001), Simulation numérique discrète de matériaux granulaires, in Micromécanique des Matériaux Granulaires, edited by B. Cambou and M. Jean, pp. 199-330, HERMES Sci. Europe, Paris.

Kuhn, M. R. (1999), Structured deformation in granular materials, Mech Mater, 31(6), 407-429.

Kuhn, M. R., and K. Bagi (2002), Particle rotations in granular materials, paper presented at 15th ASCE Engineering Mechanics Conf., Columbia Univ., New York, 1-6 June.

Luding, S. (1998), Collisions and contacts between two particles, in Physics of Dry Granular Media, NATO ASI Ser. E, vol. 350, edited by H. J. Herrmann, J.-P. Hovi, and S. Luding, pp. 285-304, Springer, New York.

Mair, K., K. M. Frye, and C. Marone (2002), Influence of grain characteristics on the friction of granular shear zones, J. Geophys. Res., 107(B10), 2219, doi:10.1029/2001JB000516.

Moreau, J. J. (1988), Unilateral contact and dry friction in finite freedom dynamics, in Nonsmooth Mechanics and Applications, CISM Courses Lect., vol. 302, edited by J. J. Moreau and P. D. Panagiotopoulos, pp. 1-82, Springer, New York.
Moreau, J. J. (1993), New computation methods in granular dynamics, in Powders and Grains 93, edited by C. Thornton, pp. 227-232, A. A. Balkema, Brookfield, Vt.

Moreau, J. J. (1994), Some numerical methods in multibody dynamics: Application to granular materials, Eur. J. Mech. A Solids, 13(4), $93-$ 114

Moreau, J. J. (1999), Some basics of unilateral dynamics, in Unilateral Multibody Contacts, edited by F. Pfeiffer and C. Glocker, pp. 1-14, Springer, New York.

Morgan, J. (1999), Numerical simulations of granular shear zones using the distinct element method: 2. Effects of particle size distribution and interplate friction on mechanical behavior, J. Geophys. Res., 104(B2), $2721-2732$.

Ngadi, A., and J. Rajchenbach (1998), Intermittencies in the compression process of a model granular medium, Phys. Rev. Lett., 80(2), 273276.

Nouguier-Lehon, C., B. Cambou, and E. Vincens (2003), Influence of particle shape and angularity on the behaviour of granular materials: A numerical analysis, Int. J. Numer. Anal. Methods Geomech., 27, 12071226 .

Oda, M. (1972a), Initial fabrics and their relations to mechanical properties of granular materials, Soils Found., 12(1), 17-36.

Oda, M. (1972b), The mechanism of fabric changes during compressional deformation of sand, Soils Found., 12(2), 1-18.

Oda, M., and K. Iwashita (1999), Mechanics of Granular Materials: An Introduction, 383 pp., A. A. Balkema, Brookfield, Vt.

Oger, L., S. B. Savage, D. Corriveau, and M. Sayed (1998), Yield conditions of an assembly of disks subjected to a deviatoric stress system, Mech. Mater., 27, 189-210.

Oron, G., and H. J. Herrmann (1998), Exact determination of force networks in a static assembly of discs, in Physics of Dry Granular Media, NATO ASI Ser. E, vol. 350, edited by H. J. Herrmann, J.-P. Hovi, and S. Luding, pp. 143-148, Springer, New York.

Peric, D., K. Runesson, and S. Sture (1992), Evaluation of plastic bifurcation results for plane strain versus axisymmetry, J. Eng. Mech., 118(3), $512-524$

Petit, J. P., and M. Barquins (1990), Fault propagation in mode II conditions: Comparison between experimental and mathematical models, in Applications to Natural Features in Mechanics of Jointed and Faulted Rocks, edited by H. P. Rossmanith, pp. 213-220, A. A. Balkema, Brookfield, $\mathrm{Vt}$

Place, D., and P. Mora (2000), Numerical simulation of localisation phenomena in a fault zone, Pure Appl. Geophys., 157(11/12), 18211845 .

Radjaï, F., and S. Roux (2002), Turbulentlike fluctuations in quasistatic flow of granular media, Phys. Rev. Lett., 89(6), 064302.

Radjaï, F., M. Jean, J. J. Moreau, and S. Roux (1996), Force distributions in dense two-dimensional granular systems, Phys. Rev. Lett., 77, 274.

Radjaï, F., D. E. Wolf, M. Jean, and J. J. Moreau (1998), Bimodal character of stress transmission in granular packings, Phys. Rev. Lett., 80(1), 6164.

Radjaï, F., J. J. Moreau, and S. Roux (1999), Contact forces in a granular packing, Chaos, 9(3), 544-550.

Radjaï, F., I. Preechawuttipong, and R. Peyroux (2001), Cohesive granular texture, in Continuous and discontinuous modeling of cohesive-frictional materials, edited by H. J. Herrmann et al., pp. 149-162, Springer, New York.

Rothenburg, L., and R. J. Bathurst (1989), Analytical study of induced anisotropy in idealized granular materials, Geotechnique, 39(4), 601614

Roux, J. N., and G. Combe (2002), Quasistatic rheology and the origin of strain, $C$. R Acad Sci. Phys, 3, 131-140.

Roux, S., and F. Radjaï (1998), Texture-dependent rigid-plastic behavior, in Physics of Dry Granular Media, NATO ASI Ser E, vol. 350, edited by H. J. Herrmann, J.-P. Hovi, and S. Luding, pp. 229 - 237, Kluwer academic publishers, Dordrecht

Roux, S., and F. Radjaï (2001), Statistical approach to the mechanical behavior of granular media, in Mechanics for a New Millenium, edited by H. Aref and J. W. Philips, pp. 181-196, Springer, New York.

Ruina, A. (1983), Slip instability and state variable friction laws, J. Geophys. Res., 88, 10,359-10,370.

Scholz, C. H. (1990), The Mechanics of Earthquakes and Faulting, 439 pp., Cambridge Univ. Press, New York.

Staron, L., J. P. Vilotte, and F. Radjaï (2002), Pre-avalanche instabilities in a tilted granular pile, Phys. Rev. Lett., 89, 204302.

Thornton, C., and G. Sun (1993), Axisymmetric compression of 3D polydisperse systems of spheres, in Powders and Grains 93, edited by C. Thornton, pp. $73-86$, A. A. Balkema, Brookfield, Vt. 
Thornton, C., and L. Zhang (2001), A DEM comparison of different shear testing devices, in Powders and Grains 2001, edited by Y. Kishino, pp. $183-190$, A. A. Balkema, Brookfield, Vt.

Troadec, J. P., and J. A. Dodds (1993), Global geometrical description of homogeneous hard sphere packings, in Disorder and Granular Media, edited by D. Bideau and A. Hansen, pp. 133-163, Elsevier, New York.

Turner, A. K. (1996), Colluvium and talus, in Landslides: Investigation and Mitigation, edited by A. K. Turner and R. L. Schuster, Spec. Rep. 247 , pp. 525-554, Transp. Res. Board, Natl. Res. Counc., Washington, D. C
Vardoulakis, I., M. Goldscheider, and G. Gudehus (1978), Formation of shear bands in sand bodies as a bifurcation problem, Int. J. Numer. Anal. Methods Geomech., 2, 99-128.

F. Bouchette, K.-J. Chang, and A. Taboada, Laboratoire de Dynamique de la Lithosphère, UMR CNRS/UMII 5573, cc 60, Université Montpellier II, F34095 Montpellier cedex 5, France. (bouchette@dstu.univ-montp2.fr; kuojen. chang@dstu.univ-montp2.fr; alfredo.taboada@dstu.univ-montp2.fr)

F. Radjaï, Laboratoire de Mécanique et Genie Civil, Université Montpellier II, F-34095 Montpellier cedex 5, France. (radjai@1mgc.univ-montp2.fr) 